\title{
Minute resolution estimates of the diffuse fraction of global irradiance for southeastern Australia
}

\author{
N. A. Engerer \\ ${ }^{a}$ Fenner School of Environment and Society, The Australian National University, Canberra, \\ Australia \\ ${ }^{b}$ National ICT Australia, Canberra Research Laboratory, Canberra, Australia
}

\begin{abstract}
Separating global horizontal irradiance measurements into direct and diffuse components has been vigorously discussed over the past half-century of solar radiation research leading to the creation of many models which attempt to compute these components with varying degrees of success. However, over the course of this discussion, nearly all studies have focused on hourly values, with no studies that have proposed a model for minute-level values of irradiance. As data-logging technologies have become much more prolific and their storage capabilities much larger, solar radiation monitoring sites are more commonly logging data at intervals much less than one hour, but no models exists that are designed to separate these measurements into direct and diffuse components. In Australia, the Australian Bureau of Meteorology and the Australian Solar Institute have compiled a dataset of tens of millions of one-minute global, direct and diffuse solar irradiance observations, comprising data from regions all around Australia. This dataset provides a unique opportunity to investigate the relationships between global irradiance and its direct and diffuse components at higher resolution than has previously been possible. Herein, the largest and most complete diffuse fraction model analysis yet undertaken for Australian solar radiation data, and the first ever to focus on minute resolution data is reported. Nine of the most prominent diffuse fraction, or "separation", models are tested against minute resolution radiation data from three datasets. The first removed cloud enhancement events in accordance with practices undertaken by the majority of studies in the literature. The second retains these events in order to assess which model would be best suited for operational purposes. The third consisted of only clear sky observations, in order to assess the performance of diffuse fraction models under clear skies. Through the course of this study only the Perez model was found to perform satisfactorily for minute resolution data at sites in southeastern Australia. Three new diffuse models proposed in this study, one trained for each of the three datasets, were found to greatly exceed the performance of existing modeling techniques, with slight improvements over
\end{abstract}

Email addresses: nicholas.engerer@anu.edu.au (N. A. Engerer), http://nickengerer.org (N. A. Engerer)

Preprint submitted to Solar Energy

March 31, 2015 
the Perez model.

Keywords: solar radiation, diffuse fraction, radiation model, cloud enhancement, validation, DNI

\section{Introduction}

The most common broadband solar irradiance measurement is global horizontal irradiance $\left(E_{g h}\right)$. This measurement records all of the solar radiation impinging on a horizontal surface as received from the skyward hemisphere, thereby including both beam (direct) radiation, which is received directly from the sun, as well as diffuse radiation, that which is scattered and reflected by atmospheric constituents and neighbouring surfaces. Most often, the horizontal beam component is referred to in its normal component form $\left(E_{b n}\right)$ also commonly entitled direct normal irradiance (DNI), whereas the diffuse radiation is only referred to in its horizontal form $\left(E_{d h}\right)$. Combining these components gives the fundamental closure equation for global horizontal irradiance, denoted as:

$$
E_{g h}=E_{b n} \cdot \cos \left(\theta_{z}\right)+E_{d h}
$$

where $\theta_{z}$ is the solar zenith angle.

Although both of these components are included in the $E_{g h}$ measurement, they must be handled separately in the modeling of solar energy applications. For example, concentrating solar power technologies that require reflection of solar radiation to a central receiver, such as those in the parabolic troughs or single tower designs, are primarily concerned with the beam component of radiation (e.g. [21]). In solar photovoltaic (PV) technologies, which are most often tilted, the radiation arriving on a non-horizontal surface cannot be determined accurately without explicit knowledge of the beam and diffuse components of radiation available, as they are modeled differently (e.g. [46]). These components must also be separated in studies sensitive to photosynthesis [54] and in the design of buildings [27], which serves to further highlight their importance.

However, directly measuring beam and diffuse components of radiation is quite costly. Beam radiation measurements are taken by a pyrheliometer device that is mounted on a sun tracker so that it can be directly pointed at the solar disc. Diffuse radiation measurements are either taken by a pyranometer fitted with a shadowball device mounted atop a sun tracker unit, or by a shadowband. These devices are expensive as they require sensitive equipment, are fitted atop moving parts and require routine maintenance, cleaning and cross-calibration [55]. Such sites can easily cost over $\$ 30 \mathrm{~K}$ USD to install. In comparison, research grade pyranometers are cheap (\$1-5K USD), and are fixed with no moving parts, thus requiring much less maintenance with a complete installation with data logger easily costing less than $\$ 5-10 \mathrm{~K}$ USD. For these reasons, horizontally mounted pyranometers have become a standard radiation measurement, and $E_{g h}$ the most readily available type of standardized solar radiation data globally. 
Yet, as previously mentioned, knowledge of the beam and diffuse components of radiation are often required. Thus, a great deal of scientific literature in the field has been focused on creating models which can separate $E_{g h}$ observations into their horizontal beam $\left(E_{b h}\right)$ and horizontal diffuse components. Therefore, it is appropriate to begin with a review of such approaches, which are referred to interchangeably in the literature as decomposition or separation models. This study will adopt the latter terminology for the duration of this work and will focus on a diffuse fraction $\left(K_{d}\right)$ modeling validation.

\subsection{Review of Available Models}

Here, the breakdown proposed by Lanini et al. [28] is adopted, introducing the $K_{d}$ models in three foundational categories: polynomial, exponential and logistic.

\subsubsection{Polynomial Models}

The majority of $K_{d}$ models operate by breaking up the relationship between $K_{d}$ and the clearness index $\left(K_{t}\right)$ into fitted polynomials in a piece-wise fashion. This approach stemmed from the relationship between $K_{d}$ and $K_{t}$ first discussed by Liu and Jordan [29] who investigated the relationship between global and diffuse irradiance at daily intervals in Blue Hill, Massachusetts. Using this relationship as applied to hourly data recorded in Canada, Orgill and Hollands [38] first suggested modeling $K_{d}$ with a piece-wise function in terms of $K_{t}$. This approach was further refined by Erbs et al. [14] for locations in the United States. Each of them requiring only $K_{t}$ as an input. For the purposes of clarity, note that $K_{d}$ and $K_{t}$ are defined as:

$$
\begin{gathered}
K_{d}=\frac{E_{d h}}{E_{g h}} \\
K_{t}=\frac{E_{g h}}{E_{\text {ext }}}
\end{gathered}
$$

where $E_{\text {ext }}$ is the horizontal component of extraterrestrial radiation

However, later research studies noted that these models did not accurately represent the spread in the $K_{d}$ for given values of $K_{t}$ (e.g. see Figure 4), and thus they began to include additional variables. Skartveit and Olseth [51], Bugler [6] and Iqbal [23] incorporated the solar altitude $(\alpha)$ in order to accommodate the shift in the distribution of $K_{d}$ values that occurs throughout a given day. Later, Reindl et al. [45] added ambient temperature and relative humidity as predictors in his $K_{d}$ model in addition to the solar altitude. Both of these modifications tended to increase the accuracy of hourly $K_{d}$ estimates.

Building on these works, numerous other polynomial based relationships have been generated for unique regions around the world including: Cordoba, Spain [43]; the Northern Mediterranean [10]; Athens, Greece [25]; India [8]; Singapore [20]; and Sao Paulo, Brazil [37]. However, as these models are less well-known and have not been widely validated, they will not be described in further detail herein. 


\subsubsection{Exponential Models}

There are two models that complete the separation through estimation of $E_{b n}$ first, rather than directly estimating $K_{d}$ as in the polynomial models. The seminal work in this area was completed by Maxwell [33] in the well-known DISC model (Direct Insolation Simulation Code), with a follow-on study extending this work completed by Perez et al. [41]. The considered approach was based on the Bird clear sky model [2], wherein the deviation from the modeled clear sky $K_{t}$ was modeled by an exponential fit according to air mass $(A M)$. The DISC model required only $K_{t}, \theta_{z}$ and $A M$ as inputs. Perez et al. [41] added to this conceptual framework by including a stability parameter, which represented the variability in the $K_{t}$ time series, and an estimate of precipitable water content $(W)$. Perez et al. [41] actually proposed two models, the first of which was a correction to the Maxwell model and the second, a simple mathematical model that allowed the conversion of global to direct beam radiation via Kasten's pyrheliometric formula [26]. Both are based on look-up tables that are broken into bins by input parameter. The Perez model is a widely used and important model, as such, it is included in this validation study.

\subsubsection{Logistic Models}

The conceptual framework for use of the logistic model in $K_{d}$ estimation was developed by Boland et al. [5] and is of particular interest to this study as it forms the basis of the current proposed minute resolution model. In their study, the authors proposed two models, one for the estimation of hourly $K_{d}$ and the other for 15 minute resolution data, developed using data from Geelong, Australia. This study represents an important step towards sub-hourly $K_{d}$ models, as it was the first to provide such estimates. The choice of the logistic function was motivated by a desire to remove the piece-wise estimations of the standard approaches. It was also the first study to use apparent solar time $(A S T)$ as a predictor variable, noting that the relationship between $K_{d}$ and $K_{t}$ was in fact asymmetrical about solar noon, thus suggesting that $\theta_{z}$ (or alternatively solar altitude) would not accurately represent this.

This initial work has been expanded upon in two additional studies, both of which focused on hourly data $[4,48]$. The first is a generalized version of the logistic function that was dependent only on $K_{t}$. The second, more recent study, developed what is commonly referred to as the BRL (Boland-RidleyLauret) model, included a total of five predictors: $K_{t}, \alpha, A S T$, a mean daily value of $K_{t}\left(\bar{K}_{t}\right)$ and a stability index $\left(\triangle K_{t}\right)$. By including the additional predictors, the BRL model was able to better predict the spread in hourly data than its predecessors. After preliminary testing, the generalized version [4] was found to have the best performance for minute resolution data, and thus is the form extensively tested herein.

Ruiz-Arias et al. [50] also used a special form of the logistic function and, after extensive review of radiation from 21 stations from Europe and North America, established a single parameter sigmoid function to calculate the $K_{d}$. They chose to exclude all extra predictor variables in pursuit of a model which 
did not require exogenous data, basing their formulation on $K_{t}$ and $A M$ only. The model is validated in the present study and is presented in Section 2.7.

\subsection{Previous Validation Studies}

To date, there have been many studies which validate the performance of the available $K_{d}$ models. Most of these validations have occurred within studies suggesting new models, much like the present study. Datasets are often regionspecific, but several more recent studies have incorporated a worldwide database for model validation (e.g [28]). The present review is best suited by dividing these studies into two groups; those that investigate model performance for hourly averages of data and those that use sub-hourly data.

When exclusively considering hourly dataset validation, the earliest validation study is that undertaken by J.W. Spencer, who validated the performance of the Bugler [6], Liu and Jordan [29] and Boes [3] models using radiation data from 12 sites in Australia [53]. A decade on, Perez et al. [41] validated three models $[44,33,14]$ while developing a new parameterized model using data from 18 sites sites in North America and Europe. They found their new model outperformed existing models, with the Maxwell model being next most accurate, followed by the Erbs model. Worth noting are the comments specific to the overestimations produced by the Maxwell model under clear, but unstable conditions. In Muneer and Munawwar [35], hourly averages of data from nine stations, located in Europe, India and Japan, were used to test and validate nine different models which contained variations of available weather and solar geometry variables. They found that the most effective modeling technique would incorporate the ratio of bright sunshine hours to day length, cloud cover fraction, and $A M$ in addition to $K_{t}$. Jacovides et al. [24] provided a very comprehensive validation of 10 models

$[8,10,14,20,25,27,31,33,37,38,46,51,52,57]$ on a single site dataset from Cyprus, but the study did not clearly rank the models in terms of their relative performance. In the development of their model, Ridley et al. [48] also validated three models $[46,51,41]$ on data from both the Northern and Southern Hemispheres, finding the Skartveit model the next most proficient after their own. The most recent study from Dervishi and Mahdavi [11] tested the ability of nine $K_{d}$ models to correctly resolve the diffuse component on hourly data recorded in Vienna. Of the models validated $[31,14,27,33,38,51,46,57]$, there were three that were selected to have superior performance: the Erbs, Reindl and Orgill and Hollands models (RMSE of approximately $40 \mathrm{~W} / \mathrm{m}^{\wedge} 2$ ).

Presently, there are four studies that have looked at the application of available models to both hourly and sub-hourly data. Boland et al. [5] developed a 15 minute resolution model, but did not test it against any other models. Ineichen [22] validated three models $[51,14,41]$ on $5,10,15,30$ and 60 minute averages of data collected from 22 sites in the United States, Europe and the Marshall Islands. For hourly data, the three models were concluded to 
have very similar precision (RMSE of $85-90 \mathrm{~W} / \mathrm{m}^{\wedge} 2$ ), with a slightly better overall performance delivered by the DirIndex model [41]. On the sub-hourly data, it was suggested that hourly models could be used without losing much accuracy, but a specific value range of this loss was not given. Lanini et al. [28] tested four models $[51,46,33,48]$ using hourly averages of data from 39 sites, discovering that the Skartveit model performed best. Additionally, a very simple validation with sub-hourly data was undertaken at a single site in Payerne, Switzerland, including some analysis of minute resolution solar radiation data. The study found that model estimates at sub-hourly time intervals have larger errors than hourly ones with Mean Bias Error and Mean Absolute Error (see section 5) losing 1.8-3.6\% and 7.6\%-10.1\% of their accuracy, respectively, as time resolutions increased from hourly to minute resolution. Finally, [18], validated 18 different separation methods with data from four U.S. sites (1-minute and 3-minute data). However, this study was not able to identify a model that performed well consistently under all-sky conditions, which provides clear motivation for future studies of this nature.

\subsection{Motivation}

The first major motivation for the present study is to further investigate the ability of currently available $K_{d}$ models to make minute resolution estimates of the diffuse fraction and propose a new model specifically designed to do so. Although both Ineichen [22] and Lanini et al. [28] have briefly commented on 5 -minute and 1-minute resolution estimates, only [18] has undertaken validation at such resolutions, and as previously mentioned, was unable to select a consistently proficient model. Further exploration and validation at these high resolutions are becoming increasingly necessary, as data storage technologies fall in cost and more frequent observations become much more plentiful. It is unknown whether the presently available suite of hourly models will provide satisfactory methods for differentiating beam from diffuse radiation at finer resolutions. For this reason, a detailed study using data from southeastern Australia is undertaken to investigate the capability of nine (see Section 2) of the most frequently used $K_{d}$ models on minute resolution data from the region.

The second motivation is to provide a new $K_{d}$ model framework that explicitly accounts for the upward climb in the diffuse fraction for $K_{t}$ values greater than 0.8. Whereas a few studies have previously discussed this phenomena (e.g. [38]), only one has directly attempted to model it [56]. Vazquez was the first to attribute this to "unshaded sun periods during partly cloudy conditions" rather than to dismiss it as anomalous and even attempted to model this region as a straight line based on the difference between $K_{t}$ and $K_{d}$ using hourly and monthly data. The present study has found the source of this increase in $K_{d}$ to be attributed to cases in which global irradiance exceeds its theoretical clear sky value. This can occur due to reflection from the sides of clouds or in cases where a thin high-level cloud deck scatters at high intensity while permitting some beam radiation to still penetrate it. Such occurrences have been termed elsewhere in the literature as "cloud enhancement" events $[9,7,32]$. In studies

related to $K_{d}$ models, cloud enhancement was first observed and commented on 
by Orgill and Hollands [38], who correctly attributed the increases in $K_{d}$ above a $K_{t}$ value of 0.8 to reflected beam radiation from clouds. That this trend was observable in their hourly dataset, and that these events occur in periods of bright sunshine, suggest that large negative biases are likely in real-world applications of these models (see [28]). However, all of the models (save the one proposed by Vazquez et al. [56]) thus far developed [38, 14, 48, 4, 50, 46] have either left these events out of their models fits, or set a fixed value for this region. Such choices are most easily attributable to the relative rarity of cloud enhancement events at the hourly time scale, thereby meaning that the previous suite of hourly models were never intended to model them. However, these events become increasingly frequent and therefore important to model, as the time resolution of the data averaging approaches zero. So, while these may be considered a second-order effect at hourly resolutions, for sub-hourly ones the importance of modeling these events is readily apparent. It is therefore worthwhile to assess the errors incurred by these models by this omission.

A third motivation is the assessment of $K_{d}$ model performance under clear skies. Diffuse irradiance can be very broadly broken into three major sources - cloud cover, the scattering and reflectance of the rest of the atmosphere and by surrounding surfaces. By selecting periods during which only clear skies are present, the behavior of $K_{d}$ models in the absence of clouds may be investigated. The available diffuse fraction during these periods is important in many applications, for example, solar energy resource assessments [47] or clear sky photovoltaic array simulations [12]. There has previously been limited validation work on clear-sky only datasets, such as in Gueymard [18] and in Gueymard [17], which have demonstrated that the best model for all-sky and clear-sky conditions cannot be assumed to be the same.

In order to properly validate existing models and develop new models suited to the purposes described, this study is completed using three separate minute resolution datasets. The first is a traditional dataset in which the cloud enhancements events are removed, the second retains them, and the third keeps only clear sky periods. The origin, quality control and selection of these datasets are detailed further in Section 3 and the development of the new models (Engerer 1,2 and 3) are described in Section 4.

\section{Separation Models Tested}

In an effort to validate the performance of existing $K_{d}$ models on minute resolution data in southeastern Australia, this study will test nine different approaches.

\subsection{Orgill and Hollands}

Orgill and Hollands [38] developed a piece-wise fitted polynomial from four years of data recorded at the Toronto Airport, comprising 12,704 hourly measurements. The independent variable for their polynomial function was the 
classical form of the clearness index $K_{t}=E_{g h} / E_{\text {exth }}$, where $E_{\text {exth }}$ is the horizontal component of extraterrestrial radiation. Their model for the $K_{d}$ took the following form:

$$
\begin{gathered}
K_{d}=1.557-1.84 * K_{t} \\
0.35 \leq K_{t} \leq 0.75 \\
K_{d}=1.0-0.249 * K_{t} \\
0 \leq K_{t}<0.35 \\
K_{d}=0.177 \\
K_{t}>0.75
\end{gathered}
$$

As a follow-on to this model, Spencer [53] re-fit the Orgill and Hollands approach to 12 sites in Australia, including four of the sites considered herein: Melbourne, Mildura, Mt. Gambier and Wagga Wagga. This is of course quite relevant to the present study and thus these modifications to the Orgill and Hollands model will also be validated according to each site. For the two additional stations, Adelaide and Cape Grim, the mean model coefficient values proposed by Spencer will be used.

\subsection{Erbs}

Noting regional differences and disagreements between existing $K_{d}$ models, Erbs et al. [14] built his own $K_{d}$ piece-wise polynomial model using global and diffuse radiation measurements from five climatologically diverse sites across the United States. Once again, the independent variable is $K_{t}$, but in this case, a fourth order polynomial was used to better fit the transitions between the three equations. The Erbs model takes the following form:

$$
\begin{gathered}
K_{d}=1.0-0.09 * K_{t} \\
K_{t} \leq 0.22 \\
K_{d}=0.9511-0.1604 * K_{t}+4.388 * K_{t}^{2}-16.638 * K_{t}^{3}+12.336 * K_{t}^{4} \\
0.22<K_{t} \leq 0.80 \\
K_{d}=0.165 \\
K_{t}>0.80
\end{gathered}
$$

\subsection{Reindl}

Reindl et al. [46] incorporated ambient temperature and relative humidity as independent variables, while keeping the three part piece-wise function form originally proposed by Orgill and Hollands:

$$
\begin{gathered}
K_{d}=1.0-0.232 * K_{t}+0.0239 * \cos \left(\theta_{z}\right)-0.000682 * T_{a}+0.0195 * R H \\
0 \leq K_{t} \leq 0.3 \quad \text { for } \quad K_{d} \leq 1.0
\end{gathered}
$$




$$
\begin{gathered}
K_{d}=1.329-1.716 * K_{t}+0.267 * \cos \left(\theta_{z}\right)-0.00357 * T_{a}+0.106 * R H \\
0.3<K_{t} \leq 0.78 \quad \text { for } \quad K_{d} \geq 0.1 \\
K_{d}=0.426 * K_{t}-0.256 * \cos \left(\theta_{z}\right)+0.00349 * T_{a}+0.0734 * R H \\
0.78 \leq K_{t} \quad K_{d} \geq 0.1
\end{gathered}
$$

\subsection{Skartveit}

The $K_{d}$ model outlined by Skartveit and Olseth [51] includes $\alpha$ as an input variable. The formulation varies between the three $K_{t}$ ranges.

$$
\begin{gathered}
K_{d}=1 \\
K_{t}<0.2 \\
f\left(K_{t}\right)=1-\left(1-d_{1}\right) *\left(0.27 * \sqrt{Q}+0.73 * Q^{2}\right) \\
Q=0.5 *\left(1+\sin \left(\pi *\left(\frac{K_{t}-0.2}{k_{1}-0.2}-0.5\right)\right)\right. \\
K_{d}=f\left(K_{t}\right) \text { for } 0.2<K_{t}<1.09 * k_{1} \\
K_{d}=f\left(K_{t}\right) \\
0.2 *<K_{t}<1.09 * k_{1} \\
K_{d}=1-1.09 * k_{1} *\left(1-f\left(1.09 * k_{1}\right)\right) / K_{t} \\
1.09 * k_{1}<K_{t} \\
d_{1}=0.15+0.43 * \exp (-0.06 * \alpha) \\
k_{1}=0.87-0.56 * \exp (-0.06 * \alpha)
\end{gathered}
$$




\subsection{Maxwell}

The Maxwell model fits the deviations from clear sky values of transmittance of beam radiation as computed by the Bird clear sky model [2] using $A M$ as the primary input parameter. It first calculates a clear sky clearness index $K_{n c}$ :

$K_{n c}=0.866-0.122 * A M+0.0121 * A M^{2}-0.000653 * A M^{3}+0.000014 * A M^{4}$

The direct beam transmittance is then computed as:

$$
K_{n}=K_{n c}-\triangle K_{n}
$$

where $\triangle K_{n}$ represents the deviation from $K_{n c}$ and is:

$$
\triangle K_{n}=a+b * \exp (c * A M)
$$

and $\mathrm{a}, \mathrm{b}, \mathrm{c}$ are polynomial functions dependent on the classical clearness index $K_{t}$.

With the transmittance estimate computed, the direct beam component is then calculated from the extraterrestrial normal radiation component:

$$
E_{b n}=E_{e x t_{n}} * K_{n}
$$

\subsection{Perez}

The Perez model utilizes a unique approach based on look-up tables for the transmittance index $X$. The input variables include the a zenith angle independent clearness index $K_{t}^{\prime}$, a variability index of $K_{t}^{\prime}$ as $\Delta K_{t}^{\prime}$, precipitable water $W$ and the solar zenith angle $\theta_{z}$. These four variables are used to retrieve a value for the transmittance index, which then is used to estimate the beam radiation via the Maxwell model (denoted DISC, see previous section, 2.5):

$$
E_{b n}=E_{b n_{D I S C}} * X\left(K_{t}^{\prime}, \theta_{z}, W, \Delta K_{t}^{\prime}\right)
$$

where $\Delta K_{t}^{\prime}$ is calculated by:

$$
\Delta K_{t}^{\prime}=0.5 *\left(\left|K_{t_{i}}^{\prime}-K_{t_{i+1}}^{\prime}\right|+\left|K_{t_{i}}^{\prime}-K_{t_{i-1}}^{\prime}\right|\right)
$$

in which i denotes the current time-step, and $\mathrm{i}+1$ and $\mathrm{i}-1$ the following and previous one, respectively.

\subsection{Boland}

The generalized version of the logistic function developed in Boland et al. [4] takes the form:

$$
K_{d}=1 /\left(1+\exp \left(-5.00+8.60 * K_{t}\right)\right)
$$




\subsection{Ruiz-Arias}

The third model from Ruiz-Arias et al. [50] is tested. It uses a quadratic dependency on $K_{t}$ and $A M$ :

$K_{d}=0.944-1.538 * \exp \left(-\left(\exp \left(2.808-5.759 * K_{t}+2.276 * K_{t}^{2}+0.125 * A M+0.013 * A M^{2}\right)\right)\right.$

with the coefficients here being those from their global model (G2) with corrected coefficients as listed in Table 4 in the 2010 publication.

\section{Data}

The data used for model validation in this study were obtained from the Australian Bureau of Meteorology's (BoM) 1-minute radiation dataset (http://reg. bom.gov.au/climate/reg/oneminsolar/index.shtml), which have recently been made available via support from the Australian Solar Institute (now part of the Australian Renewable ENergy Agency - ARENA). Each site undergoes regular calibration according to research level standards [15]. $E_{b n}$ measurements are recorded using Kipp \& Zonen CH1 pyrheliometers. Global and diffuse horizontal measurements are recorded using Kipp \& Zonen CM11 pyranometers, with a shadow ball affixed to a sun tracking unit in the latter case. In the case of the Mt. Gambier site, a Carter Scott Mk1 pyranometer was used for the earlier portion of the dataset (through March 2006). Additionally, at Cape Grim a Carter Scott DN5 pyrheliometer was in operation from March 2005 through April 2006. No empirical corrections were included for this portion of the dataset. These sites, their station number, altitude, location and modified koppen climate classification [40] are tabulated in Table 1.

\subsection{Quality Control}

First, in accordance with BoM guidance on the provided radiation data, accepted measurements were constrained to no more than $3 \%$ or $15 \mathrm{~W} / \mathrm{m}^{\wedge} 2$ of uncertainty, whichever was greater (uncertainty measures are provided for each measurement in the dataset). Next, in order to test these data more rigorously, the quality control (QC) methodology of the QCrad routine [30] was applied to the dataset before analysis. This methodology is suitable for 1-minute resolution data, and has become the standard quality control routine for the U.S. Atmospheric Radiation Monitoring (ARM) program. Only the shortwave $\left(S W_{d n}\right)$ routines were applied, as this is the only category of data tested in this study. As there were several different locations at which radiation data were collected, the configurable climatological tests were carried out with respect to the observed climatological limits of each region. This includes setting a maximum value of radiation based on the observed at each site. Long and Shi [30] provide values as computed from U.S. datasets, but do not offer any 


\begin{tabular}{cccc}
\hline Site Name & Adelaide & Cape Grim & Melbourne \\
\hline Station \# & 23034 & 91148 & 86282 \\
Elevation (m) & 2 & 95 & 113 \\
Latitude & -34.95 & -40.68 & -37.66 \\
Longitude & 138.52 & 144.69 & 144.83 \\
Data Start & Mar 2003 & Jan 2001 & Jan 2001 \\
Data End & Dec 2011 & Dec 2011 & Dec 2011 \\
Climate & CSb & Cfb & Cfb \\
\hline Site Name & Mildura & Mt. Gambier & Wagga \\
\hline Station \# & 76031 & 26021 & 72150 \\
Elevation (m) & 50 & 63 & 212 \\
Latitude & -34.24 & -37.75 & -35.16 \\
Longitude & 142.09 & 140.77 & 147.46 \\
Data Start & Jan 2001 & Jan 2001 & Jan 2001 \\
Data End & Dec 2005 & Feb 2006 & Dec 2011 \\
Climate & BSk & CSb & Cfa
\end{tabular}

Table 1: Information about the data provided by the six Bureau of Meteorology stations in southeastern Australia

explanation at how they were obtained. As it is unlikely these are representative of radiation conditions in southeastern Australia, these limits were determined herein by limiting the maximum value of each component of radiation to within a conservative 3 standard deviations above the mean observed radiation value. An example of the results from the QCrad routine using data from Adelaide in 2011 is presented in Figure 1. These images reproduce Figures 4 and 5 from Long and Shi [30].

Finally there were a few additional constraints placed on the data based on observations of the data retained after the QCrad procedure. These were based on $K_{d}$ and $K_{t}$ values. $K_{d}$ values less than 0 and greater than 1 were removed, as the diffuse fraction values exceeding the global horizontal measurement are highly suspect and their retention introduces unnecessary uncertainty into the model validation results. The $K_{t}$ lower limit was set to zero, with the upper limit dependent on the dataset under consideration (next section, 3.2). For the dataset removing cloud-enhancement events, a value of 1 was set as the maximum allowable $K_{t}$. In the dataset retaining cloud-enhancement events, a value of 1.5 was set, as this was the maximum observed value of a cloudenhancement event for zenith angles less than $75^{\circ}$. This zenith angle threshold was chosen to be consistent with those used in the QCrad routine.

\subsection{Dataset Creation}

In accordance with the experimental procedures outlined in Section 4, the radiation dataset is split into three categories. In each instance the QCrad routine was followed in accordance to the process described in the previous section, with the additional constraints then applied. 
Egh/(Ebh+Edh) Test

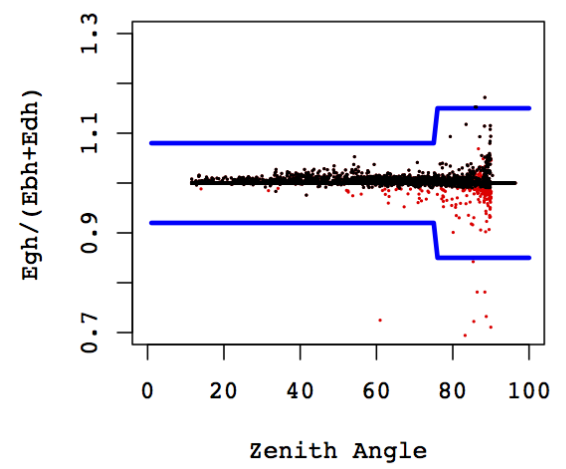

Kd Test

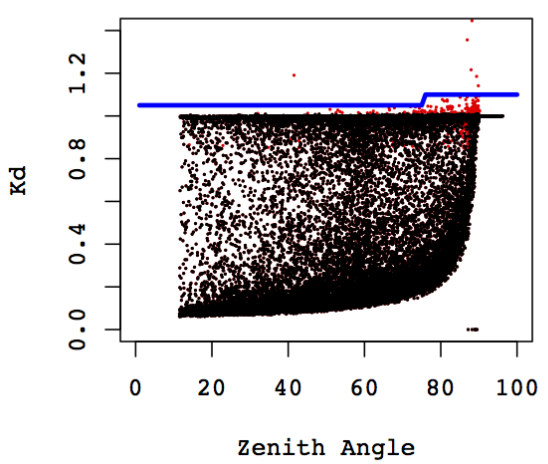

Figure 1: Two QCrad tests (Long and Shi 2006) as applied to a random selection of data from Adelaide in 2011. At left, the ratio between the observed global horizontal radiation and the sum of the diffuse and beam components is shown. Red points represent the raw data, black the filtered data and the blue lines the limits. The figure at right utilizes the same format, but instead displays the calculated diffuse fraction $K_{d}$. These figures utilize the same styling as Figures 4 and 5 from Long and Shi 2006.

The first dataset is termed the Non-Cloud Enhancement dataset (NCE). It has all periods of cloud enhancement removed by omitting all instances where $E_{g h}$ exceeds the theoretical clear-sky value. Herein, the clear sky value was determined by the REST2 model [16] as simulated based on the work outlined in Engerer and Mills [13].

The second is termed the Cloud Enhancement dataset (CE). In this dataset, the constraint based on the clear-sky model was removed, with all values of $E_{g h}$ retained.

In the third dataset, only clear sky periods were retained. In order to construct this dataset, the NCE dataset was subject to another step of postprocessing to extract clear sky periods. These were detected using the approach suggested by Reno et al. [47] as applied to observed values of $E_{g h}$. This algorithm uses the line length, slope and variability of a provided clear-sky curve to identify periods of observed data that are cloud-free. Further details on this approach can be found in Engerer and Mills [13].

An example of the results from these QC measures for $K_{d}$ at Adelaide in 2011 is presented in Figure 2. After QC processing was complete, the data were then available for model development and validation.

\section{Development of New Models}

As a result of the validation study undertaken in Section 5, it was found that hourly $K_{d}$ models were unsuitable for minute resolution data in southeastern Australia, particularly in cases where cloud enhancement events are retained. As 

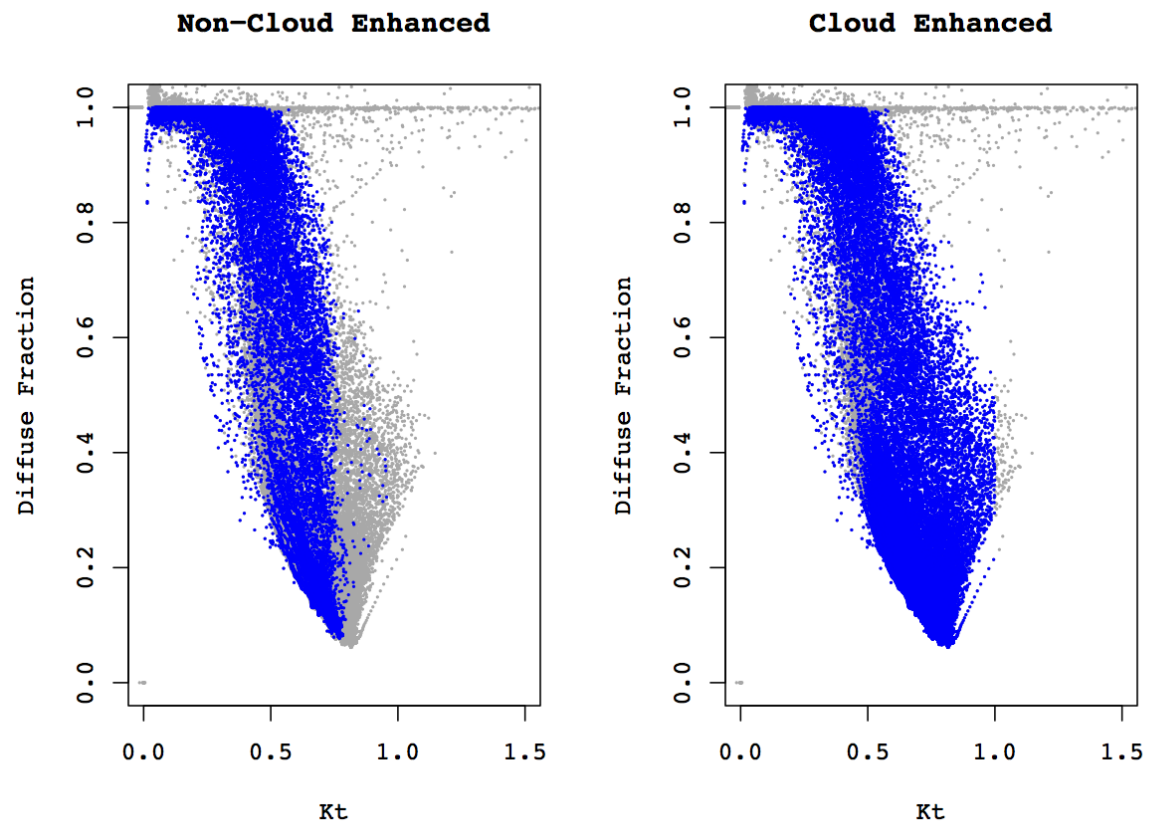

Figure 2: Minute resolution radiation data from Adelaide in 2011. Grey points include the raw observed data, with the remaining data after the QCrad routine in blue. At left, cloud enhancement events are removed by omitting all $E_{g h}$ values that exceed the clear-sky estimate from the REST2 model. In the second case, at right, the cloud enhancements are retained. 
a result, three new models were developed in this study for each of the respective datasets. The first, Engerer 1, is fit to the NCE dataset, the second, Engerer 2, is fit to the CE dataset and the third, Engerer 3, to clear sky data only. Citing the success of previous studies using the approach [4, 48], a simplified version of the generalized logistic function was used for model development:

$$
Y(x)=C+(A-C) /\left(1+\beta_{0} * \exp \left(\beta_{1}+\beta_{2} * x\right)\right)
$$

where $\mathrm{C}$ is the value of the lower asymptote, $\mathrm{A}$ the value of the upper asymptote, $\mathrm{x}$ the independent variable and the coefficients $\beta_{0}, \beta_{1}, \beta_{2}$ are determined by regression.

However, several key differences exist between the previous studies which have used this approach and the present study. The first is in the location of the lower asymptote. In the previous studies, the parameter that sets this value was either omitted or fit to a value of zero at $K_{t}=1$. However, $K_{d}$ will in no case have a value of zero during daylight periods. Even under the clearest, driest conditions, diffuse radiation will still be present due to scattering from atmospheric molecules and aerosols [26]. For this reason, the $\mathrm{C}$ parameter is retained in the regression.

The second major difference is the novel selection of the predictor variables. First and foremost, the relationship between $K_{d}$ and $K_{t}$ is included, as was first proposed in the seminal work done by Liu and Jordan [29]. Next, the $\theta_{z}$ is chosen as a predictor, based on the the important relationship between $\theta_{z}$ and $K_{d}$ first suggested in Skartveit and Olseth [51], which has been further emphasized in subsequent studies. Additionally, $A S T$ is used as the third predictor variable in order to include asymmetries in the $K_{d}$ about noon [5, 48].

To further improve the model performance, a new variable is incorporated, $\triangle K_{t c}$, which represents the deviation of the observed $K_{t}$ value from the clear sky value of the clearness index $K_{t c}$ :

$$
\triangle K_{t c}=K_{t c}-K_{t}
$$

The choice of this additional variable was motivated by two major factors. First, by the success of the "quasi-physical" DISC model [33], which is apparent in Section 5. It is referred to as quasi-physical because it directly utilizes the Bird model [2] to estimate beam radiation under clear skies before creating a fit to the deviation between observed and modeled clear sky beam radiation. This approach allows the DISC model to respond directly to cloud enhancement events (e.g. Figure 8). By using the proposed formulation of $\triangle K_{t c}$, the newly developed models can also be considered 'quasi-physical' and are directly provided information regarding cloud enhancement events as the value of $\triangle K_{t c}$ becomes negative in cases where $K_{t c}<K_{t}$.

The second major motivating factor is the strong relationship between the deviation from clear sky and the diffuse irradiance. This relationship is shown in Figure 3, where $\triangle K_{t c}$ is plotted against $K_{d}$ for both cloud enhanced and non-enhanced data. In the first (NCE) plot, as $\triangle K_{t c}$ grows, there is a clearly defined linear response in the increase of the $K_{d}$. When cloud enhancement 


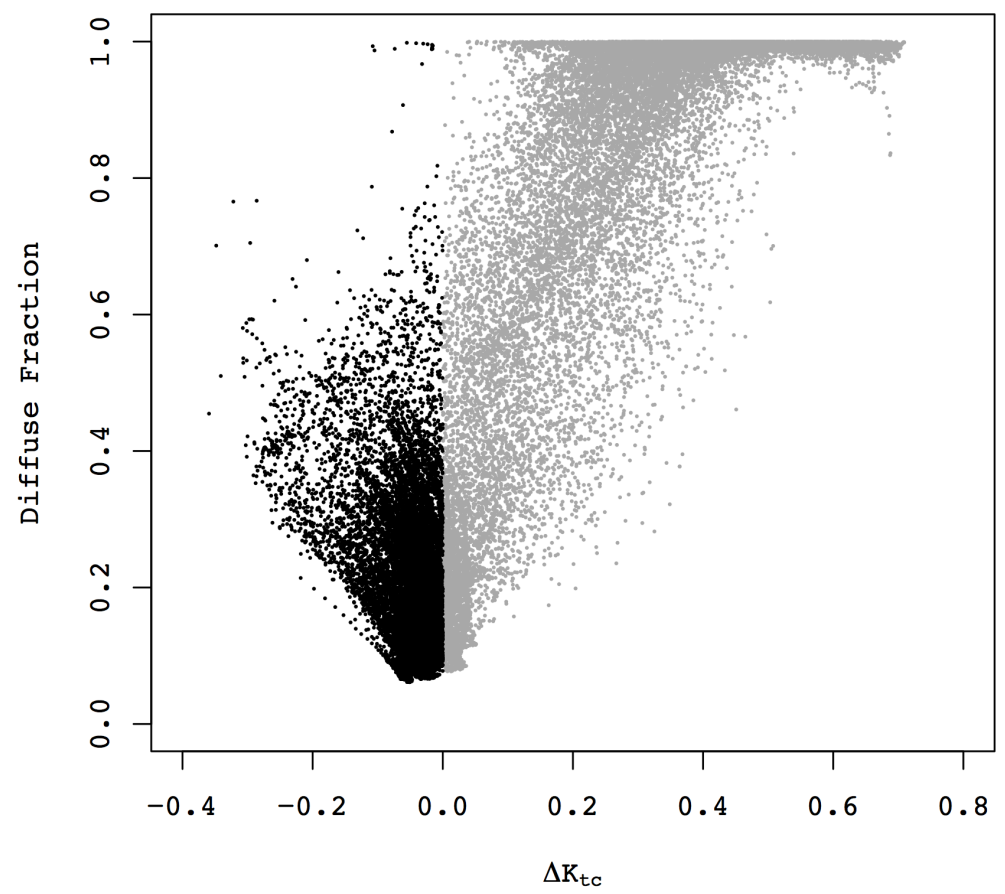

Figure 3: $\triangle K_{t c}$ is plotted against $K_{d}$. for data from Adelaide in 2011. Grey points represent calculations for all of the data (in both $\mathrm{NCE}$ and $\mathrm{CE}$ datasets) while the black shows the additional data present when cloud enhancements are retained. There is a strong linear relationship between these two variables. This relationship reverses sharply for $\triangle K_{t c}$ which are less than zero, where $K_{d}$ values start increasing as cloud enhancement events increase in intensity.

events are retained, in the second plot, the opposite becomes true. Once below zero, as $\triangle K_{t c}$ falls, there is a corresponding increase in $K_{d}$ in response to cloud enhancement events.

In this study, $K_{t c}$ is computed by the REST2 clear sky model [16]. The choice of the REST2 was based on the superior performance of the model in predicting clear sky global irradiance for sites in southeastern Australia [13]. In the case of this paper, the required information regarding atmospheric constituents (e.g. aerosol optical depth or stratospheric ozone content) was estimated using the SoDA database [49] and surface weather observations were taken from the automated weather stations co-located with the radiation sites.

By incorporating the additional $\triangle K_{t c}$ variable, along with $K_{t}$, the final form of the first model fit to the $\mathrm{NCE}$ data set is: 


$$
K_{d}=C+(1-C) /\left(1+\exp \left(\beta_{0}+\beta_{1} * K_{t}+\beta_{2} * A S T+\beta_{3} * \theta_{z}+\beta_{4} * \triangle K_{t c}\right)\right)
$$

Using combined observations from all sites, the coefficients for a "global" model were determined by non-linear least squares regression on a random selection of approximately $50 \%$ the post QC data. The resulting coefficients are presented in Table 2, and the model's performance is fully evaluated using the remaining $50 \%$ of data in Section 5.1 .

\subsection{Cloud Enhanced}

In order to create a true "all-sky" model for minute-level data, the influences of cloud enhancements must be fully considered. The primary usefulness of the aforementioned Engerer 1 model structure is as an interim means to arrive at a model which correctly captures the increase in $K_{d}$ caused by cloud enhancement events. Some modifications to the Engerer 1 model are required to accomplish this. Since the generalized logistic function is a sigmoid function, it will not rebound from its lower asymptote under any condition imposed within that functional framework. Thus, in order to create a model which was able to introduce compensatory effects, a linear correction was added to the sigmoid via a new variable, $K_{d e}$.

$K_{d e}$ is the portion of the $K_{d}$ that is attributable to cloud enhancement events and is defined by:

$$
K_{d e}=\operatorname{MAX}\left(0,1-\frac{E_{g h c}}{E_{g h}}\right)
$$

where $E_{g h c}$ is the clear sky global horizontal radiation estimate from the REST2 model.

By adding this variable as a linear correction, the $K_{d}$ model for the $\mathrm{CE}$ dataset, named Engerer 2, is:

$K_{d}=C+(1-C) /\left(1+\exp \left(\beta_{0}+\beta_{1} * K_{t}+\beta_{2} * A S T+\beta_{3} * \theta_{z}+\beta_{4} * \triangle K_{t c}\right)\right)+\beta_{5} * K_{d e}$

After incorporating this extra variable, the model was again fit by a least squares regression to the post $\mathrm{QC}$ data from each of the six sites using a random sample of approximately $50 \%$ of the post QC data. These coefficients are presented in Table 2. The model is fully evaluated using the remaining $50 \%$ of the data in Section 5.2.

\subsection{Clear-Sky Formulation}

In order to directly accommodate circumstances in which clear sky periods are of particular interest, the Engerer 1 model was also refit to the data from the extracted clear sky periods. The coefficients for the Engerer 3 model are presented in Table 2. 


\begin{tabular}{cccc} 
& Engerer 1 & Engerer 2 & Engerer 3 \\
\hline$C$ & 0.1527 & $4.2336 \mathrm{E}-2$ & 0.1090 \\
$\beta_{0}$ & -4.1092 & -3.7912 & $-2.0506 \mathrm{E}-2$ \\
$\beta_{1}$ & 6.1661 & 7.5479 & 8.1249 \\
$\beta_{2}$ & $-2.2304 \mathrm{E}-3$ & $-1.0036 \mathrm{E}-2$ & $-3.6234 \mathrm{E}-2$ \\
$\beta_{3}$ & $1.1026 \mathrm{E}-2$ & $3.1480 \mathrm{E}-3$ & $-4.1397 \mathrm{E}-2$ \\
$\beta_{4}$ & -4.3314 & -5.3146 & -5.1045 \\
$\beta_{5}$ & - & 1.7073 & -
\end{tabular}

Table 2: Coefficients for the three Engerer models. Engerer 1 is fit to the NCE dataset, Engerer 2 the CE dataset and Engerer 3 the clear sky only data set.

\section{Model Validation}

The origin of error in separation modeling is three-fold. First, there is error caused by structure of a given model and its respective ability to model the observed radiation components. Second, there is error which arises from localization of the model coefficients, which often show up as model bias. Finally, there is, of course, error in the model inputs. Thus, in order to properly evaluate the performance of these models and determine which models perform well in both regards, a total of four error measures were chosen based on those commonly present in solar radiation research literature. These are the relative Mean Bias Error (rMBE), relative Root Mean Square Error (rRMSE), Mean Absolute Percent Error (MAPE) and the coefficient of determination (R). These four measures were chosen to assess the overall bias of a model, its mean error, the error in the data points distributed about the mean and the correlation between modeled and observed output. By assessing the combined performance of these four measures using the approximately $50 \%$ of data not used for fitting of the Engerer models, the model(s) most proficient in estimating the minute resolution $K_{d}$ for southeastern Australia may be chosen.

rMBE and rRMSE are the most common error measures used in the validation of solar radiation model performance $[1,34,36,28,42]$. When both of these measures are computed, they can be used as a quantitative assessment of whether a model has acceptable performance. In order to have a "good" model, Gueymard and Myers [19] recommends an rMBE of less than 5\% and an rRMSE less than $15 \%$, whereas a "bad" model will have rMBE exceeding $10 \%$ and rRMSE greater than $20 \%$. These error measures are computed as:

$$
\begin{gathered}
r M B E=\frac{1}{n \bar{O}} \sum_{i=1}^{n} P_{i}-O_{i} \\
r R M S E=\frac{1}{\bar{O}} \sqrt{\frac{1}{n} \sum_{i=1}^{n}\left(P_{i}-O_{i}\right)^{2}}
\end{gathered}
$$

where $P_{i}$ is a given model estimate, $O_{i}$ is the observed value at time $i, n$ is the total number of observations and $\bar{O}$ is the mean over all data points. Herein, 
the validation is performed on estimates and predictions of $E_{d h}$.

To add further robustness to this analysis, two additional error measures were included. First, as a measure of the overall absolute error of a given model, MAPE was computed; and secondly, R, a measure of correlation between predicted and observed values, was computed.

$$
\begin{gathered}
M A P E=\frac{1}{n} \sum_{i=1}^{n}\left|\frac{P_{i}-O_{i}}{O_{i}}\right| \\
R^{2}=1-\frac{\sum_{i=1}^{n}\left(O_{i}-P_{i}\right)^{2}}{\sum_{i=1}^{n}\left(O_{i}-\bar{O}_{i}\right)^{2}}
\end{gathered}
$$

\subsection{Non Cloud Enhanced Validation}

The analysis commenced with the NCE dataset, by producing model estimates using data from the Adelaide station from the 2011 calendar year (only the testing data points were used; not training data). These are presented in Figure 4. The Adelaide station was chosen in order to provide a detailed intercomparison of model performance due to it being the most thoroughly tested Australian site amongst the various studies in the literature [53, 5, 48, 39]. In each of these plots, the observed $K_{d}$ is plotted against $K_{t}$ in grey as computed from the quality controlled radiation data with cloud enhancements removed. Model estimates of $K_{d}$ values are calculated and plotted as points in blue.

The differences between single and multi-variate models are immediately apparent. Predictions from the Orgill, Spencer, Erbs and Boland models appear as a single line, while the Reindl, Skartveit, Maxwell, Ruiz-Arias and Engerer 1 models include many possible $K_{d}$ values for each value of $K_{t}$. The general shape of the predicted versus observed values appears to agree best in the Skartveit, Maxwell, Perez and Engerer 1 models, but all save the Engerer 1 model show a rebound from low $K_{d}$ values at $K_{t}$ values exceeding $\sim 0.75$. As the overall precision cannot be inferred from this figure alone, the predicted versus observed values correlations are also investigated. These are plotted against each other in Figure 5. These are are revealing, with positive biases at low $K_{d}$ apparent in the Orgill, Spencer, Erbs and Skartveit models, and a negative bias in the Reindl and Ruiz-Arias models in these regions (the Ruiz-Arias model appears to have an overall negative bias for all values of $K_{d}$ ). The correlations are best for the Skartveit, Reindl and Engerer 1 models, with R values of 0.86, 0.88 and 0.93 , respectively.

Also of interest is an investigation into how the models concerned perform throughout the day. In order to assess this, the MAPE and rRMSE values are calculated and plotted as a function of $\theta_{z}$ in Figures 6 and 7 with error calculations broken up into $\theta_{z}$ bins of 2.5 degrees. Only bins with at least $0.5 \%$ of the total available data were accepted into this analysis, so as to ensure each bin contained a representative sample. In terms of MAPE values, model performance throughout the day shows an overall gradual decrease in model error with increasing $\theta_{z}$. The MAPE scores of the the Engerer 1 and Perez are comparable (15-25\%), with the Maxwell model showing similar results for $\theta_{z}$ 


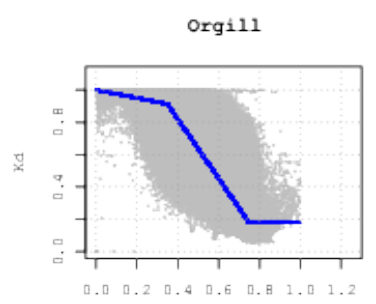

Kt

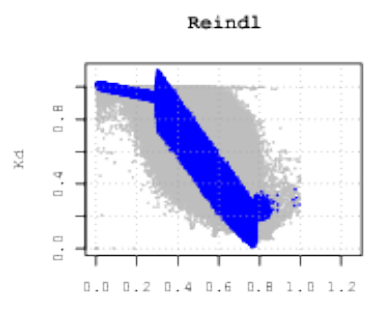

xt

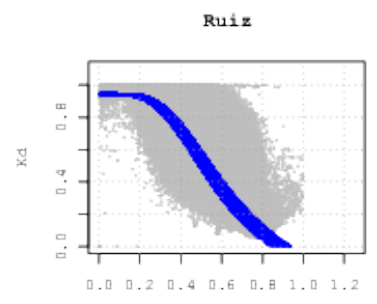

$\mathrm{Kt}$

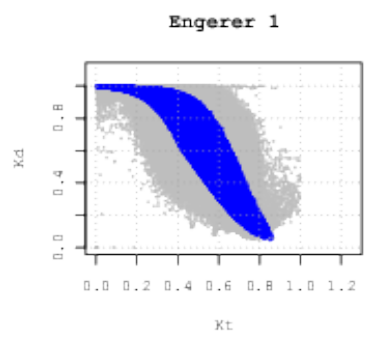

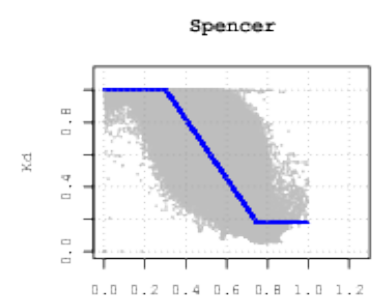

$\mathrm{xt}$

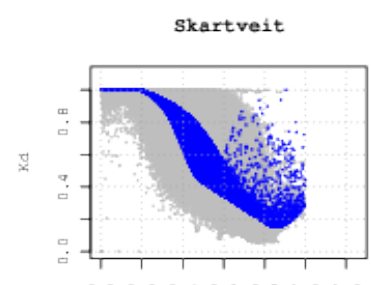

Kt

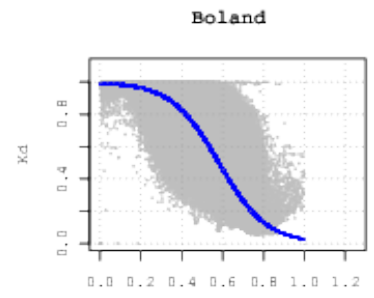

Kt

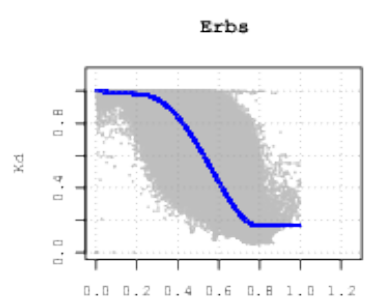

Kt.

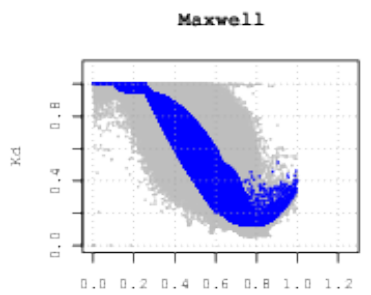

kt.

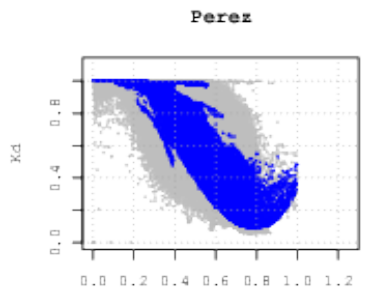

Kt

Figure 4: $K_{d}$ versus $K_{t}$ plots for all ten $K_{d}$ models. In each plot, grey points represent the post QC data as recorded at Adelaide in 2011 with cloud enhancement events removed. The model estimates are shown in blue. 

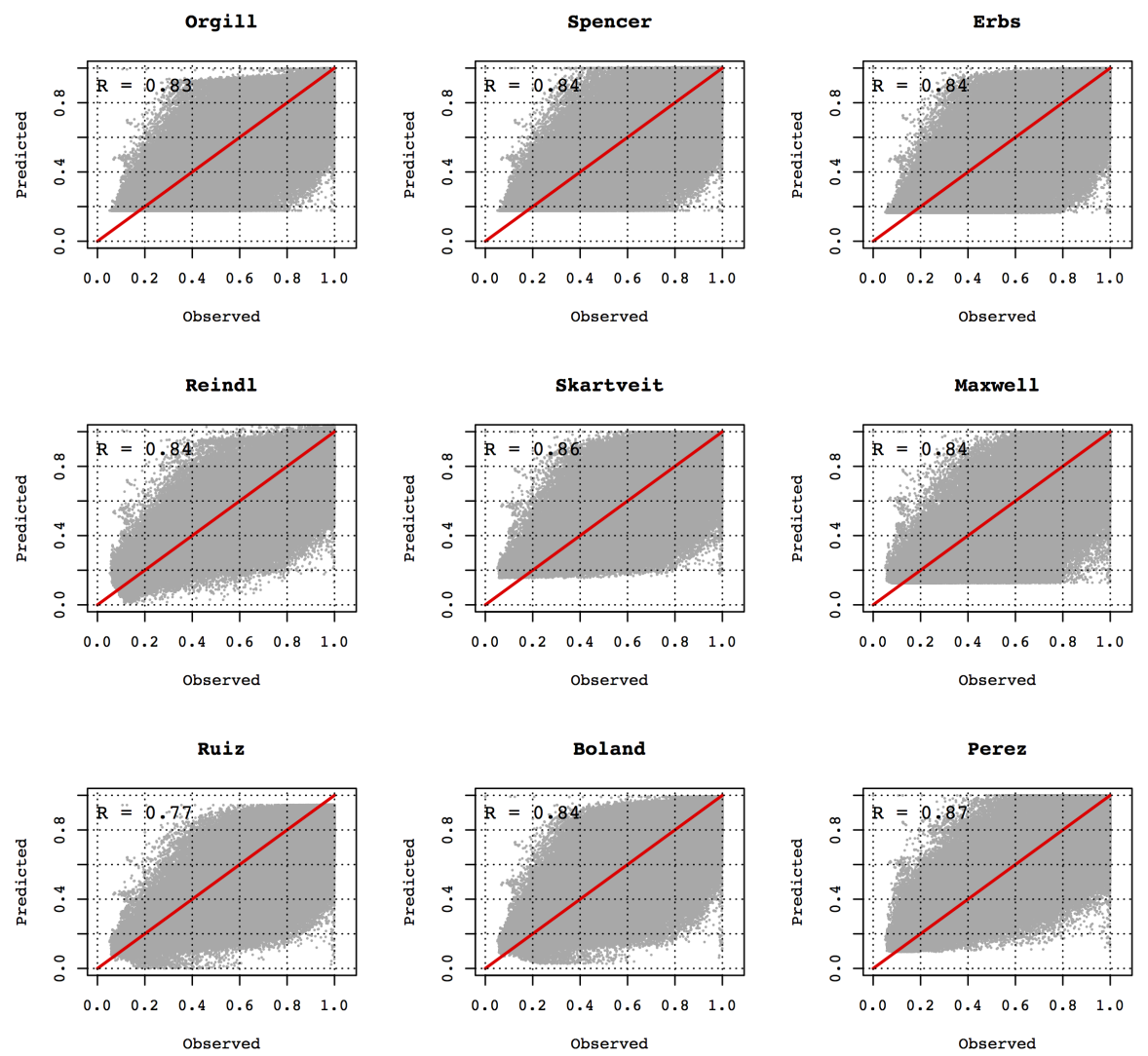

Engerer 1

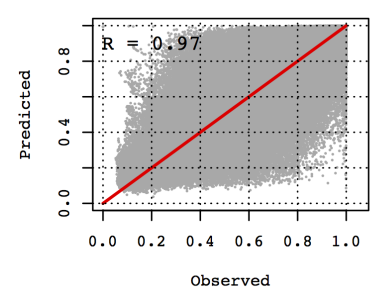

Figure 5: Predicted versus observed values of $K_{d}$ for each of the ten models as computed for 2011 data from Adelaide. Also included is the the coefficient of determination as R. 
greater than 45 degrees. The Reindl model does the poorest overall in terms of absolute error, with the Orgill, Spencer, Ruiz-Arias and Boland models with MAPE consistently in the $25-30 \%$ range.

The rRMSE results are quite consistent, with a slight overall decreasing trend with increasing $\theta_{z}$. The Engerer 1 and Perez model have indistinguishably similar accuracy and a clear advantage over the remaining models. The Skartveit model is clearly the third most accurate model. The Orgill, Spencer and Erbs models also share very similar results, with the Ruiz-Arias model a clear outlier and the worst overall accuracy except possibly for extreme $\theta_{z}$ values. The convergence of all models with very high $\theta_{z}$ is also noted.

It is of interest to note that the upward trend in model error with decreasing zenith angle is the opposite of that found in other separation model validation studies $[28,24,11]$. As previous studies have been completed with hourly data, this suggests that modeling undertaken at sub-hourly (namely minute level) data resolutions experiences a different error relationship with solar zenith angle than has previously been found. Cloud enhancement events are one obvious explanation for this change (Section 5.2), but that alone cannot fully explain this phenomenon, as the relationship change exists in the present dataset where cloud enhancements have been removed. Further investigation is needed to understand why this is so.

In addition to these figures from Adelaide, results from a much more comprehensive analysis are available in Table 3. This table was constructed by evaluating all ten models (including the new model, Engerer 1) at each of the six stations using the four selected error measures. Over 30 million data points were available to the analysis with at least 3 million at each site.

Mean diffuse radiation measures were found to be higher for areas that are cloudier and more temperate (Adelaide, Cape Grim, Melbourne, Mt. Gambier) versus those in more arid areas (Mildura and Wagga). All relative error measures are presented with respect to these mean values.

In analysis of the rMBE results, the overall trend in the models is an overprediction of $K_{d}$ at Melbourne and Mildura, with underprediction at the remaining sites. The Maxwell and Ruiz-Arias models are observed to have a negative bias at all stations, while the bias becomes positive for the Orgill, Erbs, Reindl and Skartveit models at Mildura. The Engerer 1 model has the lowest rMBE values overall (with the exception of a notable overprediction at Mildura) which was expected, given that the model was built on a dataset which is assumed to be representative of that used for validation.

The variations in mean diffuse radiation between sites are reflected in the MAPE values, which tend to increase as the mean value falls. For this reason, larger MAPE values tend to be observed at Mildura and Wagga and the lowest at Mt. Gambier. MAPE values tended to be in excess of $20 \%$ for nearly all models and all sites, with the exception of the Engerer 1 model at which scored below $20 \%$ at all sites except Mildura. The Perez model scored below 20\% at Cape Grim, Mt. Gambier and Wagga, indicating a slightly increased absolute error over the Engerer 1 model overall. The remaining models have less consistent results, with the Reindl, Spencer and Orgill models tending to have the largest 


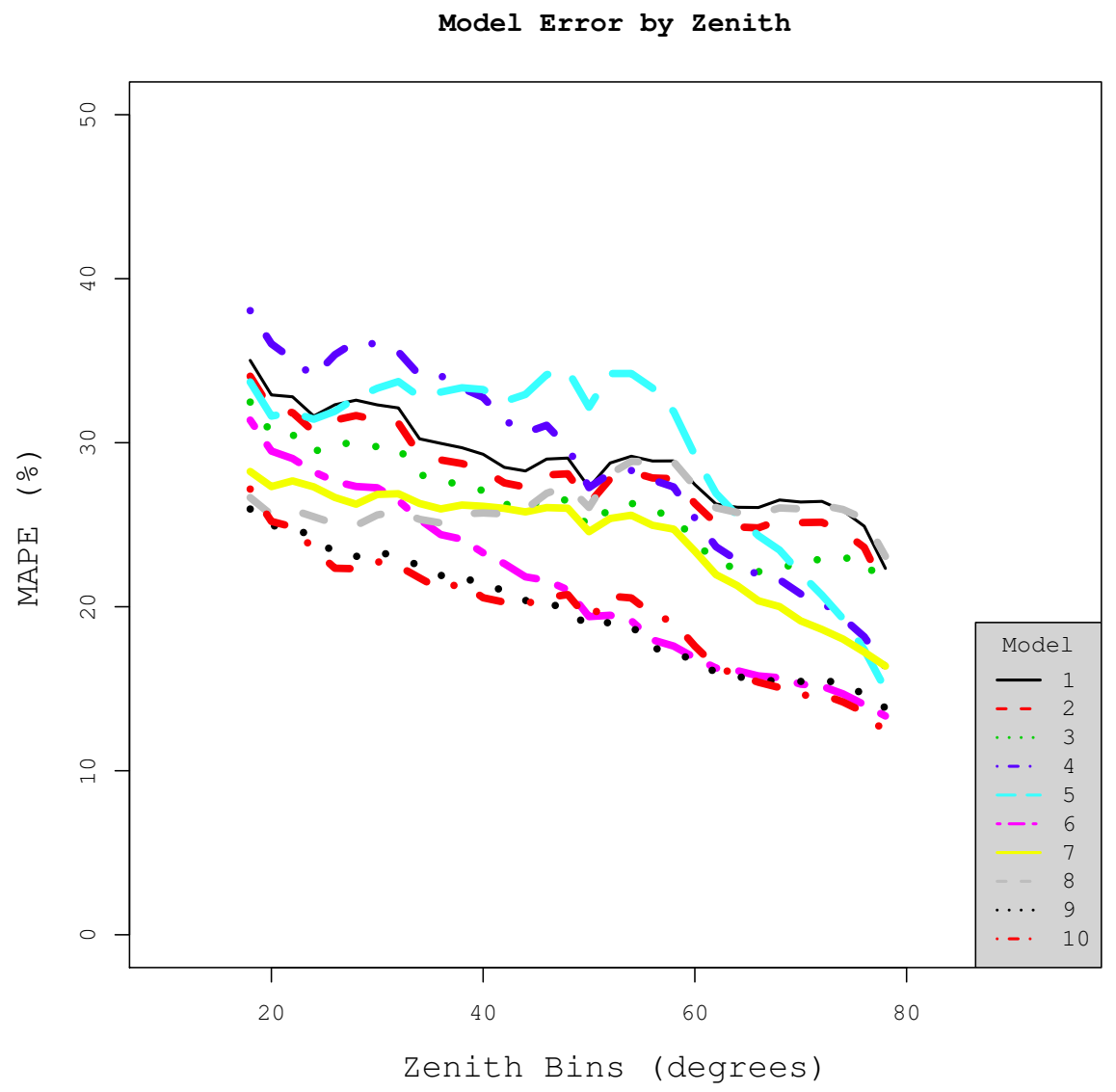

Figure 6: Model MAPE values as a function of $\theta_{z}$. The models correspond to the following numbers: 1 Orgill, 2 Spencer, 3 Erbs, 4 Reindl, 5 Skartveit, 6 Maxwell, 7 Ruiz-Arias, 8 Boland, 9 Perez, 10 Engerer 1. 
Model Error by Zenith

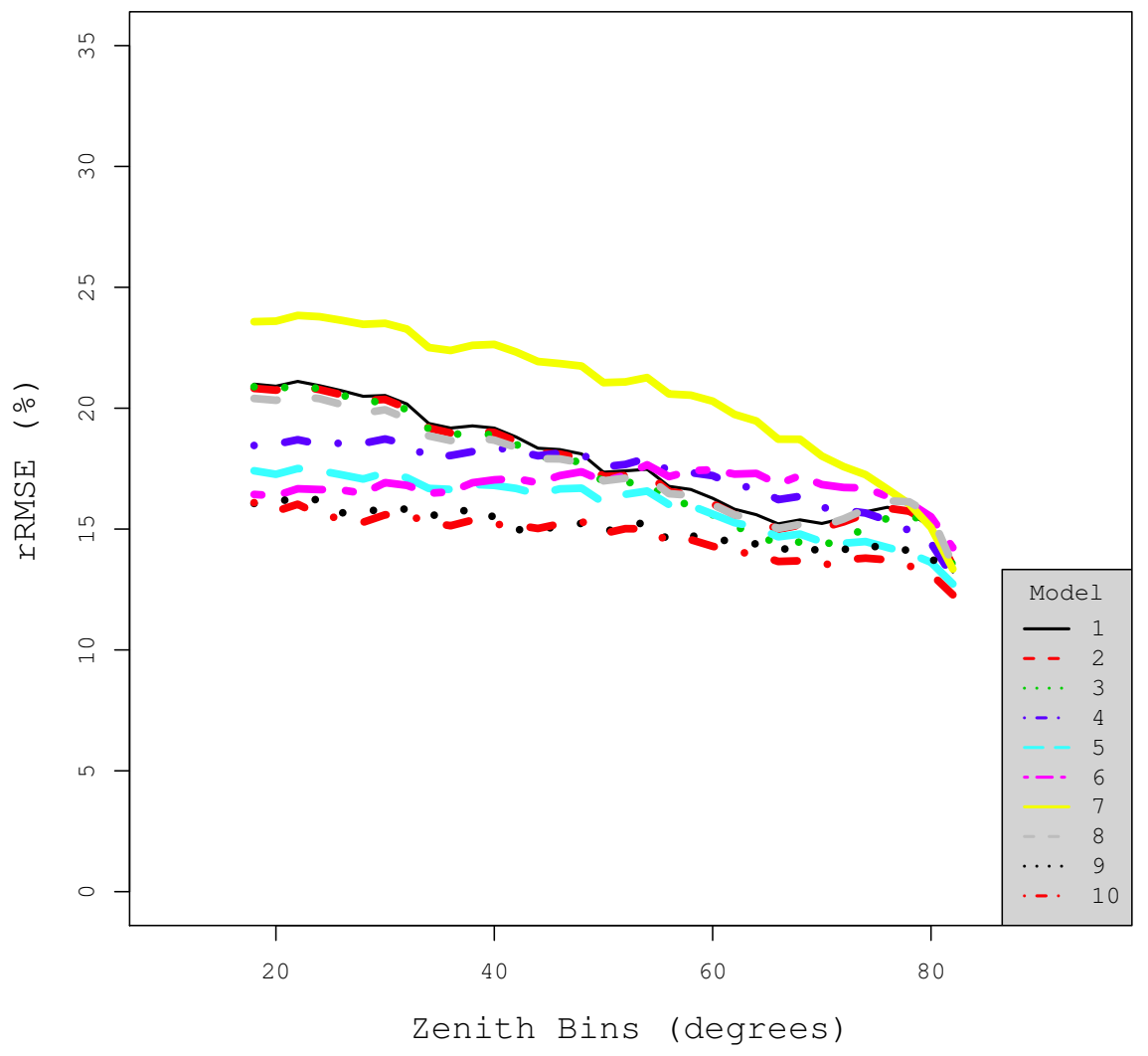

Figure 7: As in Figure 6, but for rRMSE. 
MAPE.

The rRMSE and $R$ calculations are measures of the "distance" between a model's predicted value and the observed value and thus are good measures of overall accuracy. Whereas a locally fit model will most often experience lower MAPE and rMBE values, this it not necessarily true of the rRMSE. The Engerer 1 and Perez models consistently scores higher $R$ and lower rRMSE values at all stations, with all remaining models having rRMSE values greater than $15 \%$ and $R$ values below 90 . The least accurate model overall appears to be the Spencer model, which could be considered surprising as it was originally fit to Australian data. Also of interest are the changes in results with the Ruiz-Arias and Boland models after receiving re-fit coefficients (again, denoted by*). The Ruiz-Arias model shows consistent improvements, with rRMSE values falling by as much as $5 \%$. Changes in the accuracy of the Boland model improvements were notably unremarkable. The Engerer 1 and Perez models clearly demonstrate they are the superior models for one minute data, with an overall slight advantage in the Engerer 1 model results. These were followed in accuracy by the Skartveit and Maxwell models.

With this analysis of the values provided in Table 3, the best models for estimating $K_{d}$ of minute resolution solar radiation in southeastern Australia under non-cloud enhanced conditions are the Engerer 1 and Perez models, which have "good" results (Gueymard and Myers [19] rMBE $<5 \%$ and $\mathrm{rRMSE}<15 \%$ ) for many of the sites tested.

\subsection{Cloud Enhanced Validation}

The analysis for the CE data set adopts the same approach undertaken in the previous section, using Adelaide as a point of inter-comparison.

Figure 8 presents calculations for each model in a manner equivalent to that of Figure 4, but with the cloud enhancements retained. Perhaps the most immediate difference is the upturn in the modeled values of $K_{d}$ that appear at $K_{t}$ values greater than $\sim 0.6$. Five of these models (Orgill, Spencer, Erbs, Ruiz-Arias and Boland) do not respond to the increased observed $K_{d}$. The remaining four have varying levels of response, with the Maxwell, Perez and Engerer 2 models appearing to perform best and the Skartveit model appearing to be a bit too vigorous with the cloud enhancement events. However, in order to properly assess which of the four models makes the best predictions in this case, further analysis is needed. For that purpose, Figure 9 provides plots of predicted versus observed values of $K_{d}$. As was noted in Section 5.1, several of the hourly models systematically overestimate $K_{d}$ at low values of observed $K_{d}$, with the Ruiz-Arias model appearing to underestimate for all values of $K_{d}$. The Engerer 2 model and Perez models, conversely, have much more even distributions about the identity line, and the respective $R$ values of 0.93 and 0.9 are the highest of those tested. The Skartveit and Maxwell model also perform well, both with an $R$ value of 0.88 .

Further insight can be drawn from the MAPE and rRMSE plots as a function of $\theta_{z}$, which show strong contrasts to the analogous plots presented in Figures 6 and 7. First, in Figure 10, MAPE values are greatly increased at lower $\theta_{z}$ 


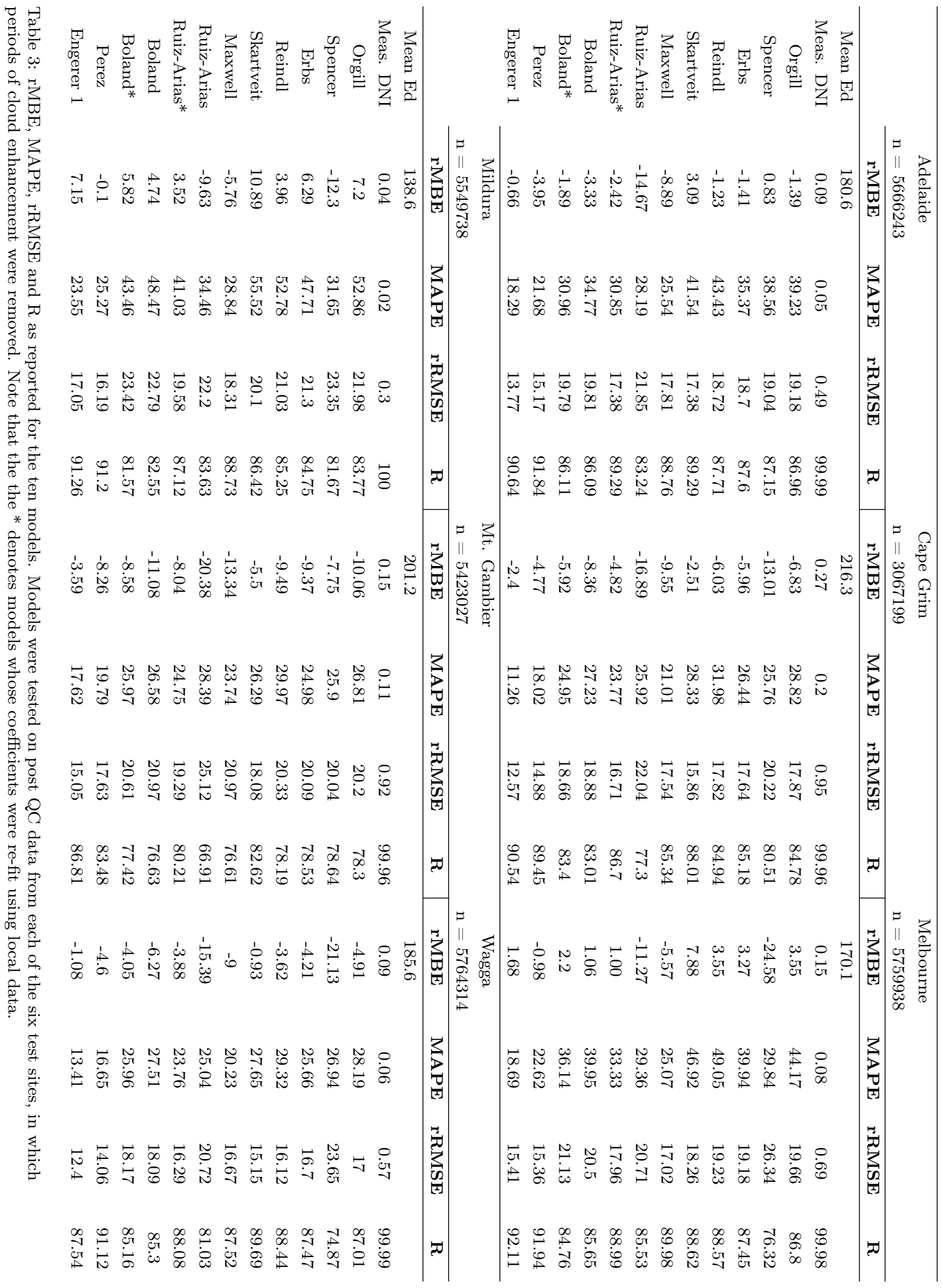




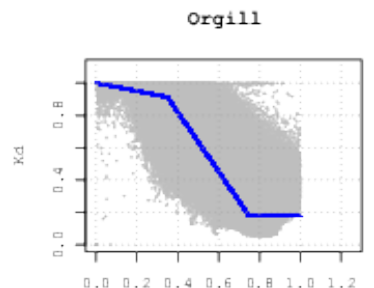

$\begin{array}{llllllll}0.0 & 0.2 & 0.4 & 0.6 & 0.8 & 1.0 & 1.2\end{array}$

$\mathrm{Kt}$

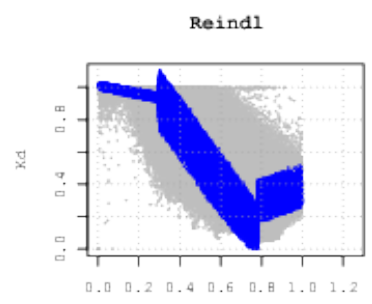

xt.

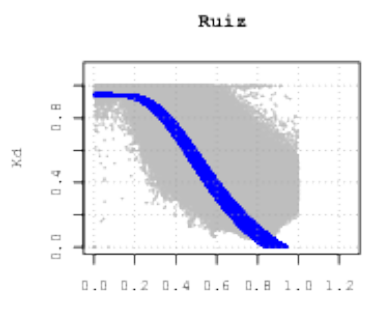

xt

Engerer 2

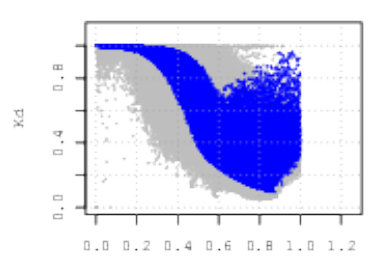

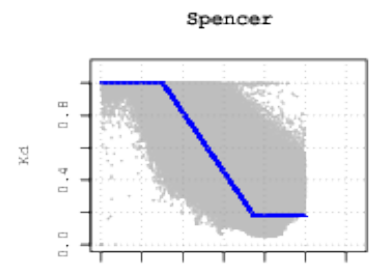

$\begin{array}{lllllllllll}0.0 & 0.2 & 0.4 & 0.6 & 0.8 & 1.0 & 1.2\end{array}$

Kt

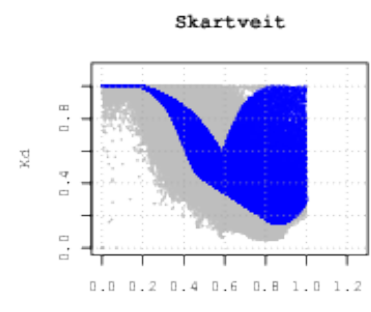

$\mathrm{Kt}$

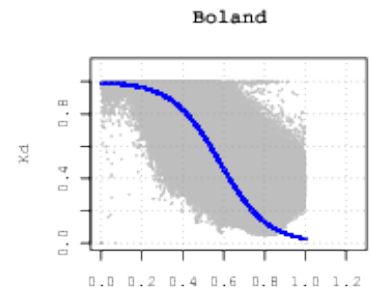

$x \mathrm{t}$

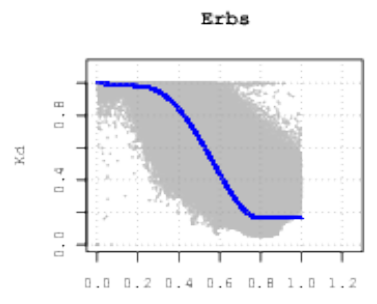

Kt
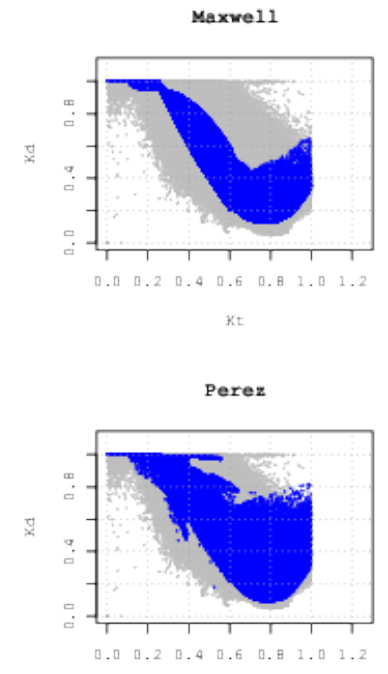

Figure 8: As in Figure 4, but with cloud enhancement events retained. 

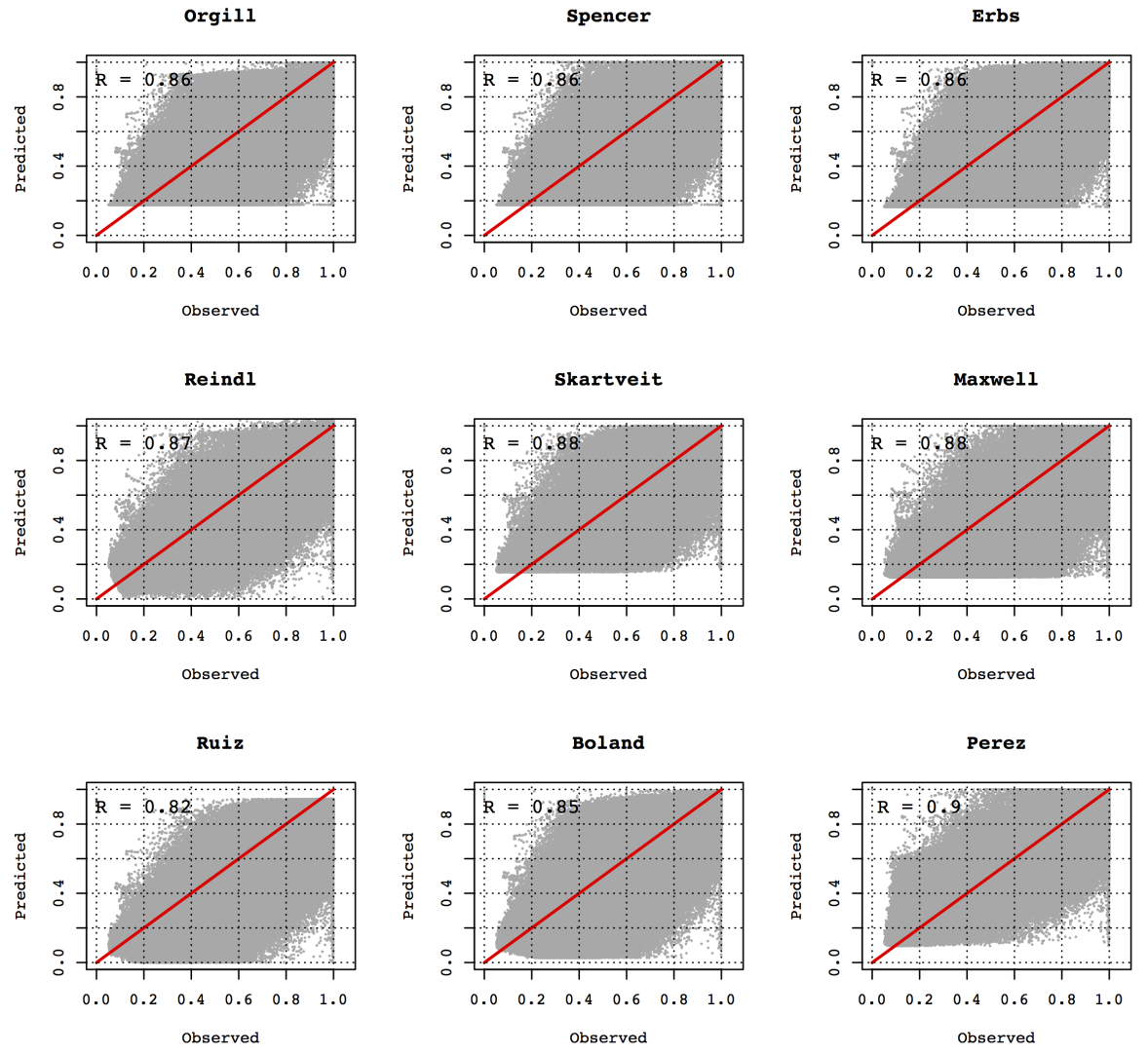

Engerer 2

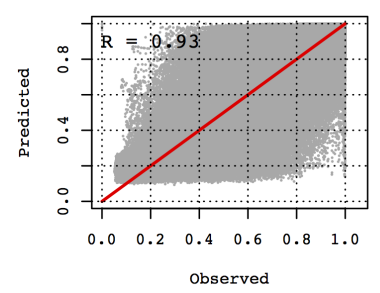

Figure 9: As in Figure 5, but with cloud enhancement events retained. 
when the sun is higher in the sky, and fall throughout the day. The change in this plot is attributable directly to the inclusion of cloud enhancement events. As such, it can be inferred that during lower $\theta_{z}$ periods the influence of cloud enhancement events is greater, as the models which do not attempt to estimate them do particularly poorly. The Engerer 2 and Maxwell models have the best MAPE values overall.

In terms of the rRMSE plot in Figure 11, large rRMSE issues are apparent with the Ruiz-Arias model while the Engerer 2 and Perez models maintain the best accuracy at all $\theta_{z}$. There is very similar and consistent accuracy amongst the univariate Orgill, Spencer, Erbs and Boland models. Model RMSE scores throughout the day, are overall, quite consistent with a slight trend toward reduced errors with increasing $\theta_{z}$.

Table 4 presents the overall result from model evaluation on the CE dataset. There is a general increase in the relative and mean errors over the previous suite of results (Section 5.1) for those models that do not include considerations for the increase in $K_{d}$ originating from cloud enhancement events (Orgill, Spencer, Erbs, Ruiz-Arias, Boland). The strongest bias is in the Ruiz-Arias model which was already apparent in the previous Figures $8 \& 9$, which consistently underpredicts $K_{d}$ by more than $9 \%$. This bias disappears once the re-fit coefficients are considered, however, indicating the model structure is not the origin of the error. Results across the six sites and remaining models are notably inconsistent, but it is clear that overall the Perez, Engerer 2 models have the least biased results, with the Skartveit, Maxwell and Boland the next least biased grouping. This suggests that the apparent upturn in the Perez, Maxwell and Skartveit models for increased $K_{t}$ may indeed be accounting for cloud enhancement events.

The Engerer 2 and Perez models are the only models to receive MAPE values less than $20 \%$ at any site. The Engerer 2 consistently has lower MAPE scores than the Perez model, by as much as $6.7 \%$ (at Cape Grim). In the remaining models, MAPE values are in the $20-40 \%$ range, with several instances of values exceeding $50 \%$. This is indicative of the increased challenges present when attempting to estimate $K_{d}$ during cloud enhancement periods. The Maxwell model stands out from the remaining models in the MAPE values, with all results in the $21-28 \%$ range. Of note, the Skartveit model was one of the worst performers when absolute error is considered, despite having had relatively good rMBE scores.

rRMSE and $R$ values clearly show the Engerer 2 and Perez models have the best accuracy, followed by the Maxwell model. Several of the models fall into the "bad" category [19], with rRMSE values exceeding $20 \%$ at some of the sites. These are the Ruiz-Arias, Spencer, Erbs, Reindl and Boland models. However even the new model formulation in Engerer 2 fails to meet the "good" category for rRMSE $(<15 \%)$ values at some of the sites (Melbourne, Mildura, Mt. Gambier), which is indicative of the challenge of including and modeling cloud enhancement events.

Overall, the Engerer 2 model will provide the best minute resolution $K_{d}$ estimates from global horizontal measurements in southeastern Australia under 


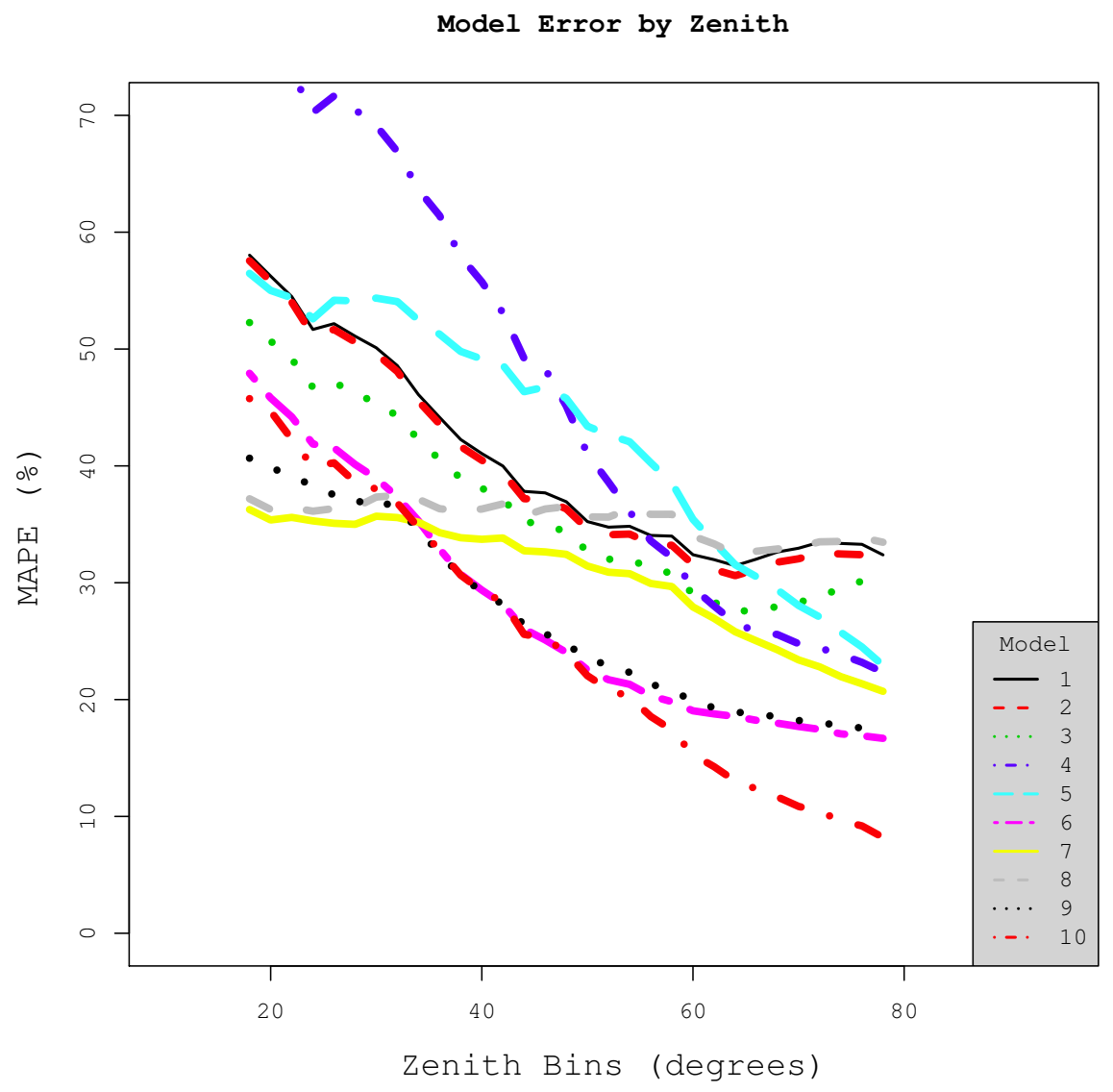

Figure 10: Model MAPE values as a function of $\theta_{z}$ for cloud enhanced data. The models correspond to the following numbers: 1 Orgill, 2 Spencer, 3 Erbs, 4 Reindl, 5 Skartveit, 6 Maxwell, 7 Ruiz-Arias, 8 Boland, 9 Perez, 10 Engerer 2. 
Model Error by Zenith

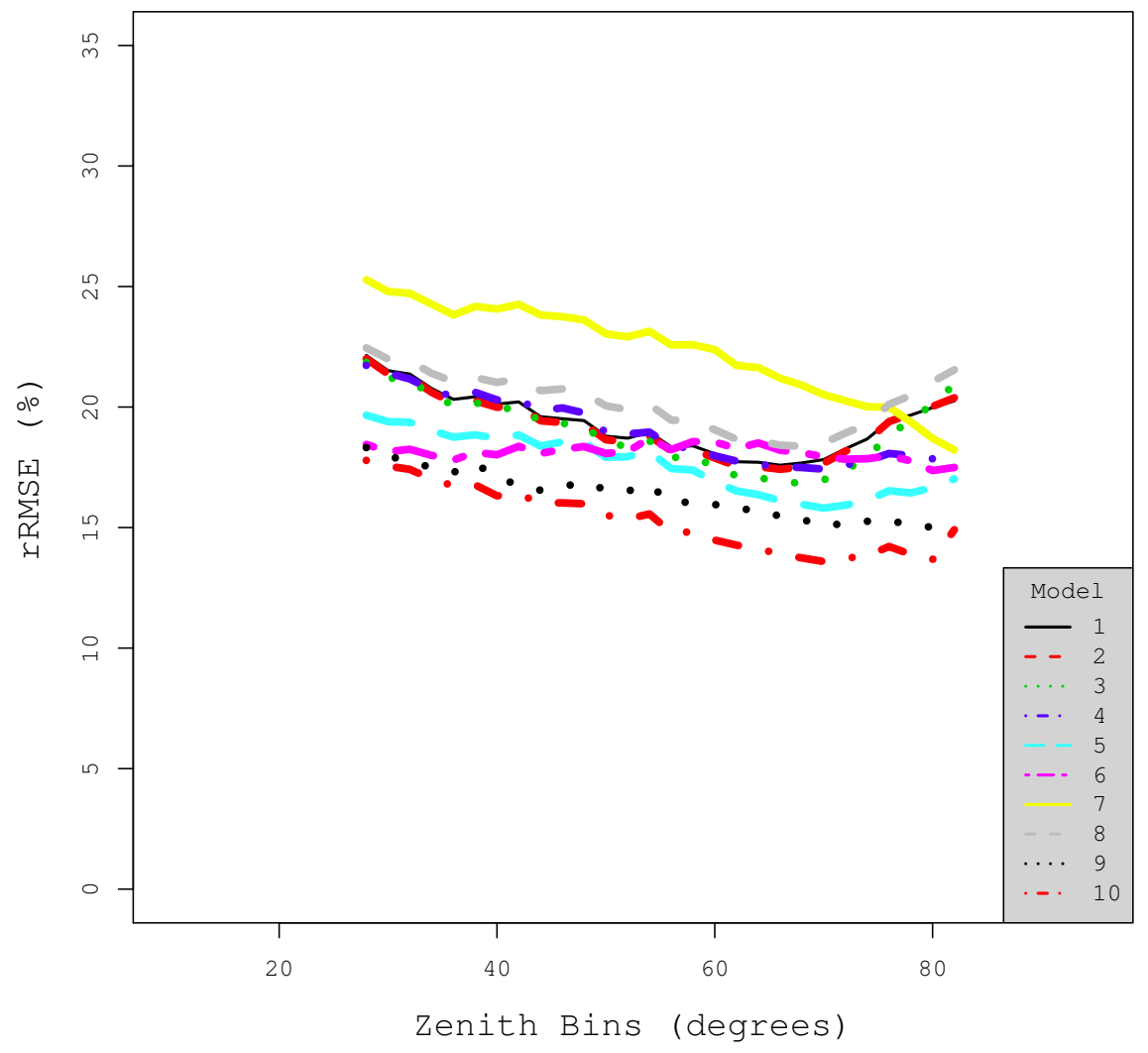

Figure 11: As in Figure 10, but for rRMSE values. 
real-world, cloud enhanced conditions. The Perez model has nearly equivalent performance, but with slightly increased rMBE, MAPE and RMSE values. The Maxwell model also does well overall in this cloud enhanced dataset, but the Skartveit model's initial promise was clearly misleading once MAPE, RMSE and $\mathrm{R}$ are considered.

\subsection{Clear Sky Validation}

Finally, the clear sky dataset was evaluated following the same approach given in Sections 5.1 and 5.2. Figure 12 has the model predictions presented as in Figure 4. Model estimates from Orgill, Spencer, Erbs and Boland all show systematic over-estimation of $K_{d}$ for $K_{t}$ values greater than 0.4 , while the Skartveit model appears to have an overall fairly poor fit to the data. The Engerer 2, Perez and Maxwell models have a better fit, but produce an upward turn in $K_{d}$ values during clear-sky periods, which is erroneous as cloud enhancement events were removed by the clear sky filtering process [13]. Figure 13 provides additional insight. Again, here the over-estimation of $K_{d}$ by the Orgill, Spencer, Erbs and Boland models is even more apparent, earning them relatively low $R$ scores in the 0.83 - 0.84 range. The Maxwell, Perez and Engerer 2 models appear to do quite well under clear sky situations $(0.91-0.93)$, but the Engerer 3 model does best with an $R$ value of 0.94 and the most balanced correlation (with respect to the identity line).

Model error as a function of $\theta_{z}$ reveals very large MAPE values for most models at low $\theta_{z}$ (Figure 14). With the exception of the Ruiz-Arias, Boland and Engerer 3 models, MAPE values climb above $60 \%$ for $\theta_{z}$ less than 40 . Even at increasing $\theta_{z}$, MAPE values above $40 \%$ are routine. Figure 15 shows significant contrasts to the previous similar figures in Section 5.1 and 5.2, with rRMSE trends with increasing $\theta_{z}$ being mixed. The Boland and Ruiz-Arias models both see rRMSE climb with increasing $\theta_{z}$, while the Orgill, Spencer, and Skartveit models first fall and then rise. Overall, there is a significant spread in the model error values, with the Reindl model performing least well. Here one can clearly see the benefit of having a model fit to clear sky occurrences, as the Engerer 3 model maintains the most consistent rRMSE score, falling below $20 \%$ for $\theta_{z}$ greater than 45 degrees. Both the Maxwell and Perez model have comparable performance to the Engerer 3 model for $\theta_{z}$ greater than approximately 55 degrees, but clearly the Engerer 3 clear sky trained model is showing itself to be relatively useful for use under clear sky conditions.

In Table 5, the results from all models at all sites are presented. In addition to the Engerer 3 model, the operational Engerer 2 model is tested. An analysis of the rMBE values shows many large positive biases in the tested suite of models, particularly at Mildura, which is the most arid of the regions tested. Several models standout with lower rMBE values, including the Maxwell, Perez, Engerer 2 and Engerer 3, which was the least biased model. Also notable, the Ruiz-Arias model appears to have benefited from its previous under-prediction of $K_{d}$ with relatively small rMBE values present.

Given that the dataset has now been reduced to clear sky instances, the mean values of diffuse radiation are reduced. Values are now approximately $\frac{1}{3}$ 


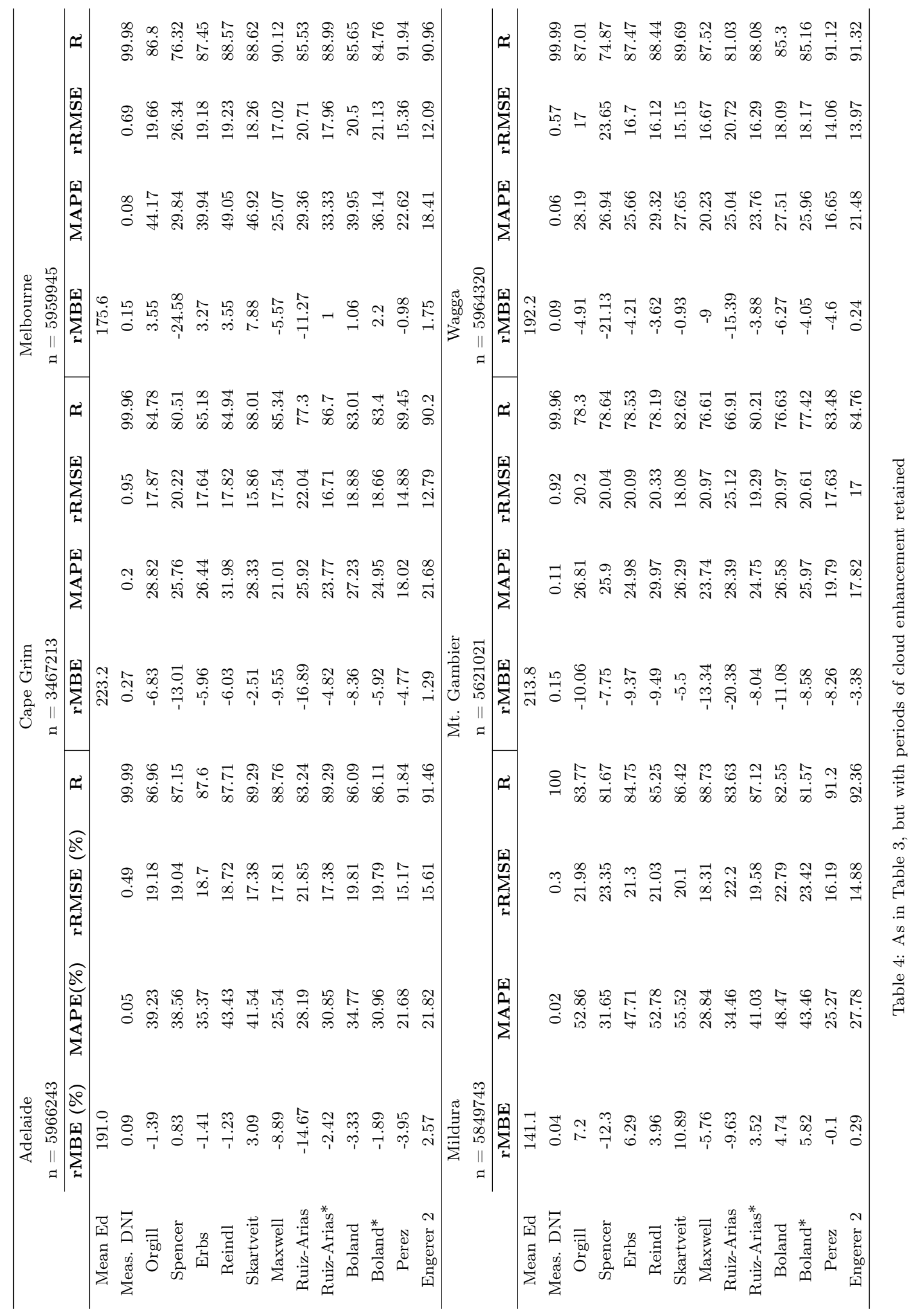




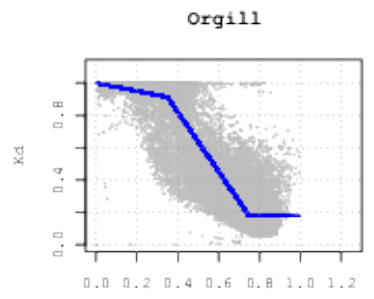

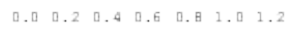

$\mathrm{Kt}$

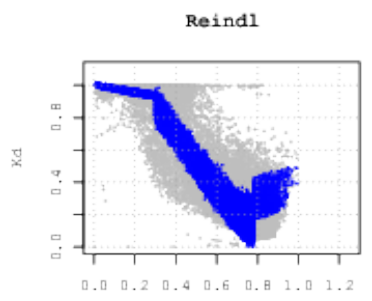

kt.

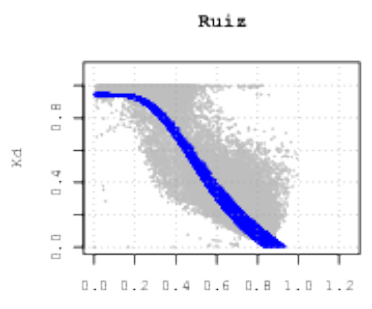

$x \mathrm{t}$

Engerer 2

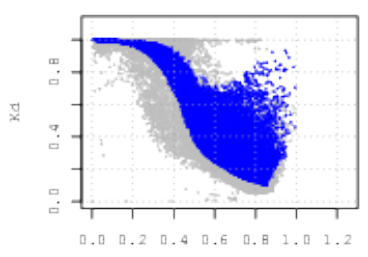

kt

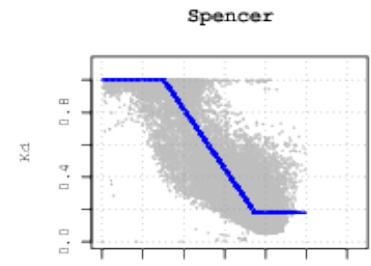

$\begin{array}{lllllllllll}0.0 & 0.2 & 0.4 & 0.6 & 0.8 & 1.0 & 1.2\end{array}$

Kt
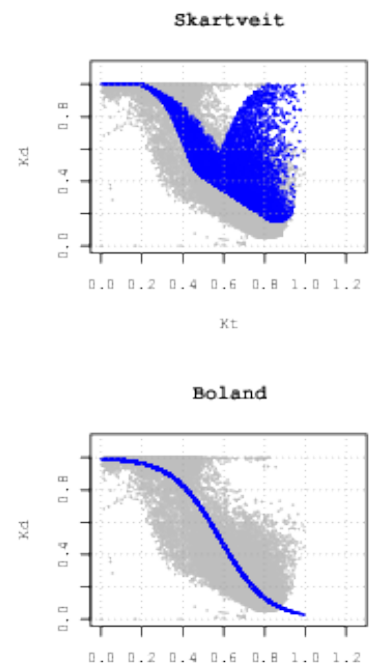

Engerer 3

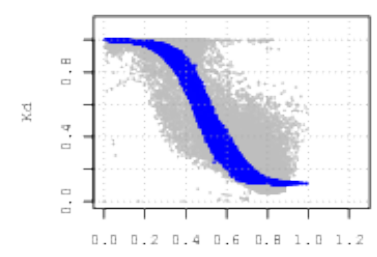

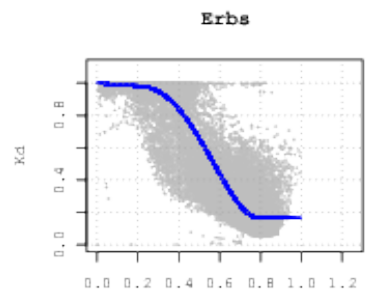

Kt
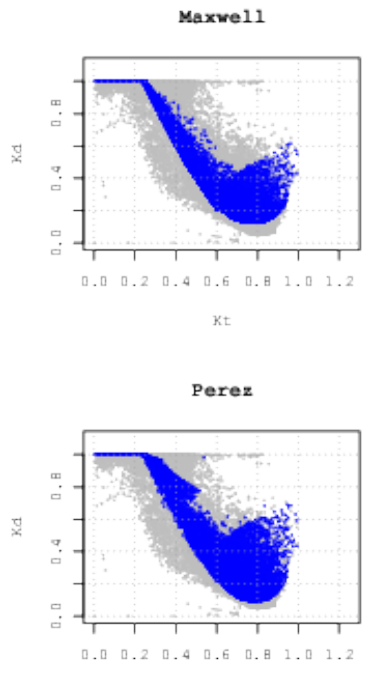

Figure 12: As in Figures 4 and 8, but for clear sky periods only. 

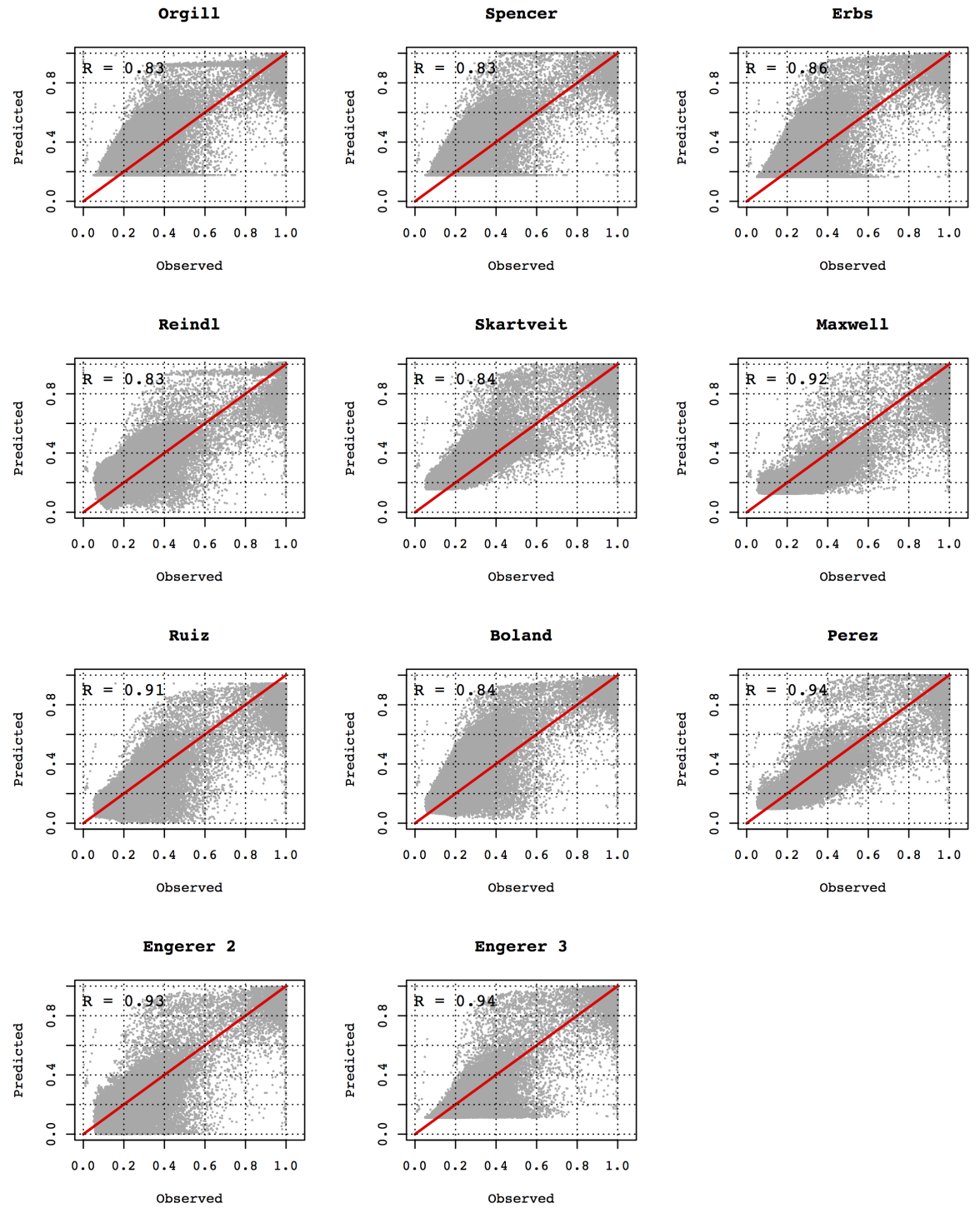

Figure 13: As in Figures 5 and 9, but for clear sky data only. 


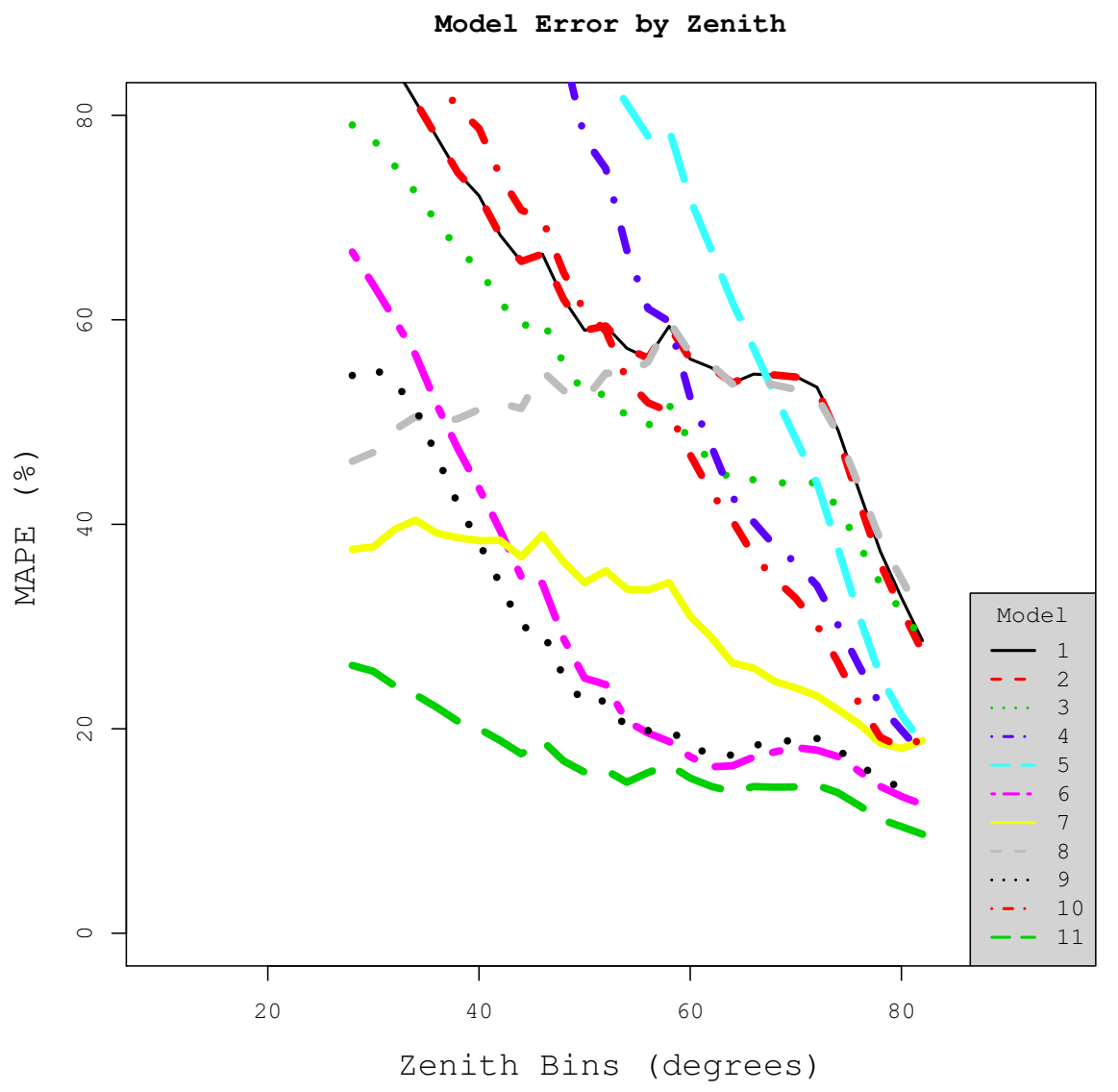

Figure 14: Model MAPE values as a function of $\theta_{z}$ for clear sky data. The models correspond to the following numbers: 1 Orgill, 2 Spencer, 3 Erbs, 4 Reindl, 5 Skartveit, 6 Maxwell, 7 Ruiz-Arias, 8 Boland, 9 Perez, 10 Engerer 2, 11 Engerer 3. 
Model Error by Zenith

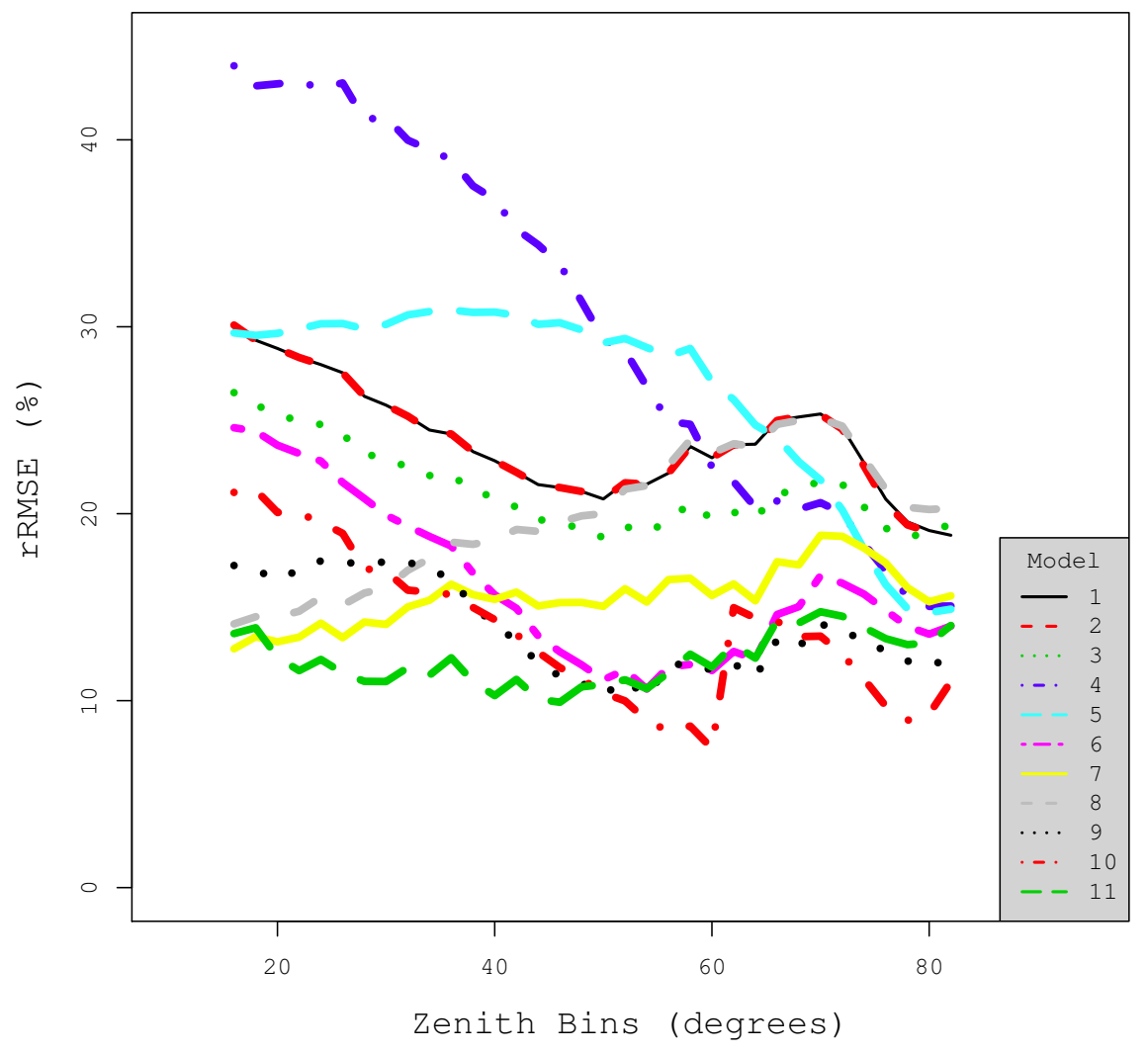

Figure 15: As in Figure 7, but for clear sky data. 
of their all-sky values, meaning that MAPE values are likely to be much higher than in previous cases. This is clearly the case, with MAPE ranging from 9.36 - 78\%. In terms of the MAPE results, no particular model was found to have consistently larger absolute errors, but the clear sky trained Engerer 3 model does routinely offer improvements over the next best models - Perez and Engerer 2 .

There is not a great deal of difference between models in terms of rRMSE values. Which generally fall in the 10-25\% range across all sites and all models (with a few exceptions). The clear sky trained Engerer 3 model does provide slight improvements in accuracy overall, but this is not always the case (e.g. Perez model at Melbourne has slightly, but not significantly, higher accuracy). Once the $R$ values are considered, the generation of a clear sky specific Engerer 3 model does appear justified and useful, as it provides increased performance across all metrics considered. However in the absence of a clear sky trained model, the Perez model proves itself as quite valuable once again, and has "good" performance for estimating the clear sky diffuse fraction (Gueymard and Myers [19] $\mathrm{rMBE}<5 \%$ and $\mathrm{rRMSE}<15 \%$ ). But is is important to recall that the Perez model absolute error grows very quickly once $\theta_{z}$ values fall below 40 degrees.

\section{Conclusion}

In order to validate the performance of nine of the most common diffuse fraction models in southeastern Australia, minute resolution radiation data from the Australian Bureau of Meteorology's radiation sensor network were retrieved from six locations and split into three datasets. One with cloud enhancements removed, another with them retained and a third for clear sky periods only each of them extensively quality-controlled in accordance with current practice in the literature [30]. In response to the apparent inability of these models to generate consistently accurate and precise estimates under the various conditions imposed by these three datasets, three new models have been developed one for each set of conditions. The new models utilize a logistical function framework and include a new variable, $\triangle K_{t c}$, which is the deviation from the clear sky value of $K_{t}$ as calculated by the REST2 model. In the cloud-enhancement model, a second new variable is included to provide a linear correction, $K_{d e}$, which accounts for the portion of the diffuse fraction resulting from cloud enhancement events. These models are designated as the Engerer 1, Engerer 2 and Engerer 3 models, for the non-cloud enhanced, cloud-enhanced and clear sky cases, respectively. The Engerer 1 model's primary purpose was as the basis for the development of the Engerer 2 model. As has been shown, cloud enhancement events are quite common in minute level data datasets, so in practice the Engerer 1 model is not actually useful in the real-world context. This is, instead, the role of the Engerer 2 model, which is an "all-sky" model suitable for use on sub-hourly data, wherein cloud enhancements should be explicitly modeled.

By testing these new models against nine models representative of the state of the art in hourly separation modeling, it has been made clear that the new 


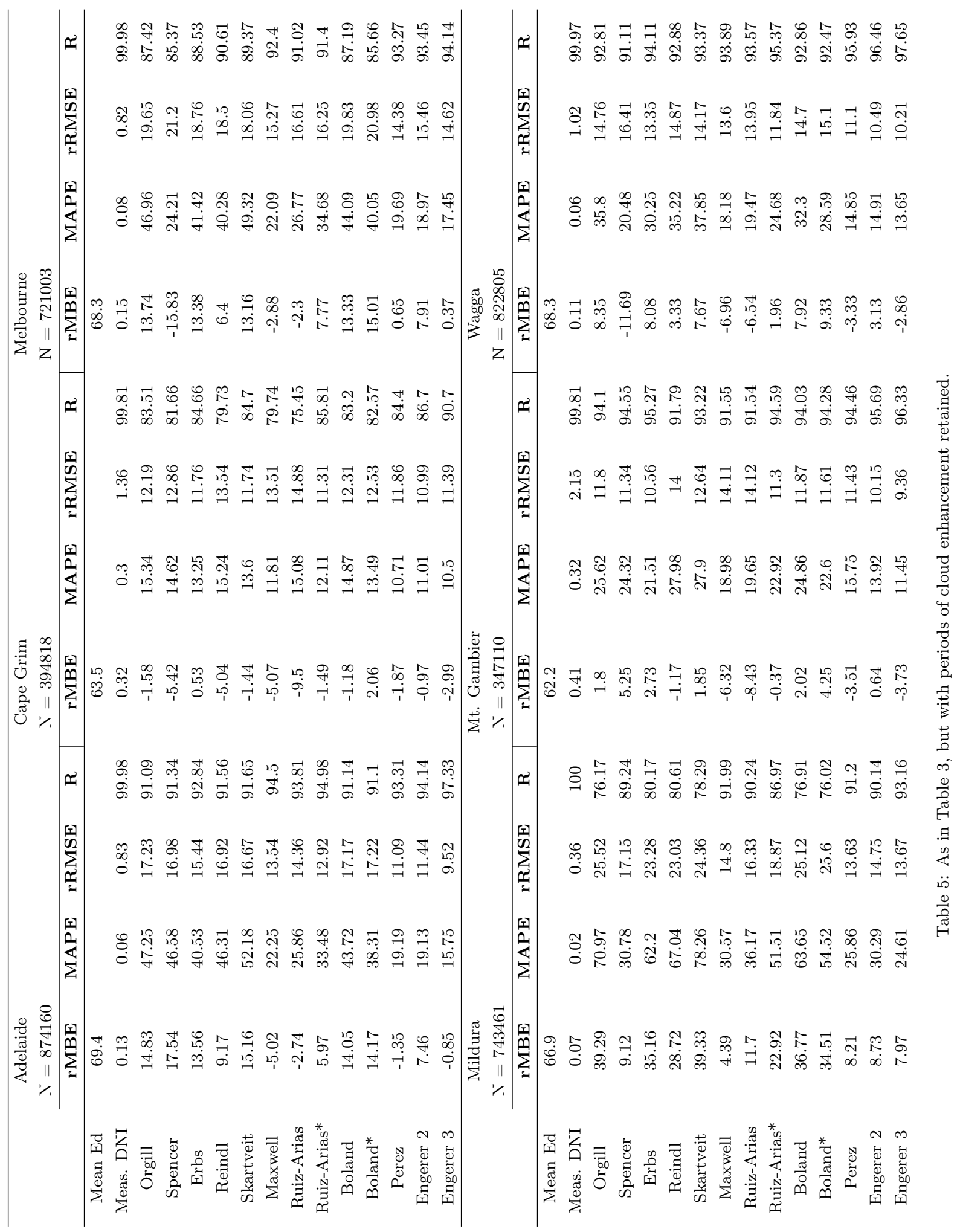


models offer more precise and accurate performance in southeastern Australia, but further validation will be require to determine the usefulness of their coefficients globally. This has been demonstrated through the evaluation of four error measures (rMBE, MAPE, rRMSE, $R^{2}$ ). This clearly suggests, but does not prove, that models fit to minute-resolution data are in fact better suited to make minute-resolution estimates of the diffuse fraction. All three models are capable of being used in real-time (operationally), in that all of their input values can be obtained in real-time (e.g. no information from future time steps required).

In addition to the development and validation of the new models, several additional important conclusions can be made. First, the Perez model is a clear stand out from the other eight models being tested, achieving "good" performance $(\mathrm{rMBE}<5 \%$ and $\mathrm{rRMSE}<15 \%$ ) for the non-cloud enhanced and clear sky datasets in the bulk error measurements. It also scored well at most $\theta_{z}$ values in all three categories, and was competitive with the newly fit Engerer 1 and Engerer 2 rRMSE values. For this reason, it is a conclusion of this manuscript that the Perez model is quite suitable for use on 1 minute resolution radiation data within Australia, with the expectation that this likely to be true in other regions. This is a novel finding as compared to Gueymard [18] which was unable to select a superior model from the 18 models tested.

Secondly, in terms of a real-world operational model, the ability to handle cloud enhancement events is quite important, as they are increasingly common and significant as the averaging interval of the observed data decreases. Only four models demonstrated the capability to model the cloud enhanced region of the $K_{t} K_{d}$ relationship. These were the Perez, Maxwell, Skartveit and Engerer 2 models. After the detailed analysis undertaken herein, the Perez, Maxwell and Engerer 2 models were found to model this region competently (matching the shape of the $K_{t} K_{d}$ relationship, achieving rMBE scores of $<10 \%$ and rRMSE scores of $15-20 \%$ ), which is an important finding. Still, it is important to note that with falling $\theta_{z}$ values, MAPE scores grow quickly, exceeding $40 \%$ error for $\theta_{z}<30$ degrees.

Another conclusion is that a clear sky trained diffuse radiation model is quite useful, as demonstrated by the superior performance of the Engerer 3 model over the other ten models tested, which was particularly noticeable for $\theta_{z}$ values greater than 40 degrees (during the brightest hours of sunlight). Future work might attempt to divide datasets into clear and cloudy conditions in real-time, using a different separation model for each condition, as they are fundamentally different in terms of radiation phenomena.

Finally, the author would like to announce that an $\mathrm{R}$ package which will allow for the replication of these results, including the REST2 model simulation and newly introduced Engerer 1-3 models will be published to the author's website in mid 2015. The validation datasets will also be made available. This will allow for future collaboration and additional testing, as well as future tuning of the Engerer model coefficients, if required. 


\section{Acknowledgments}

The author would like to acknowledge the contributions of the Australian Bureau of Meteorology and the Australian Solar Institute (now the Australian Renewable ENergy Agency) to this study, specifically in the provision of the one minute level solar radiation data. Additionally, thanks are extended to National ICT Australia (NICTA) and the United States National Science Foundation Graduate Fellowship Research Program, both of which have partially funded this research. Users who are interested in obtaining the code required for computing these models, or the curated datasets tested herein, may obtain them from the author's website.

\section{References}

[1] Badescu, V., Gueymard, C. a., Cheval, S., Oprea, C., Baciu, M., Dumitrescu, A., Iacobescu, F., Milos, I., Rada, C., Jul. 2013. Accuracy Analysis for Fifty-Four Clear-Sky Solar Radiation Models Using Routine Hourly Global Irradiance Measurements in Romania. Renewable Energy 55, 85103.

[2] Bird, R., Hulstrom, R., 1981. A Simplified Clear Sky Model for Direct and Diffuse Insolation on Horizontal Surfaces. Tech. rep., Solar Energy Research Institute.

[3] Boes, E., 1979. Insolation Modeling Overview. Energy 4, 523-525.

[4] Boland, J., Ridley, B., Brown, B., Apr. 2008. Models of Diffuse Solar Radiation. Renewable Energy 33 (4), 575-584.

[5] Boland, J., Scott, L., Luther, M., 2001. Modelling the Diffuse Fraction of Global Solar Radiation on a Horizontal Surface. Environmetrics 12, 103116.

[6] Bugler, J., Jan. 1977. The Determination of Hourly Insolation on an Inclined Plane Using a Diffuse Irradiance Model Based on Hourly Measured Global Horizontal Insolation. Solar Energy 19 (5), 477-491.

[7] Cazorla, A., Olmo, F. J., Alados-Arboledas, L., Jan. 2008. Development of a Sky Imager for Cloud Cover Assessment. Journal of the Optical Society of America. 25 (1), 29-39.

[8] Chandrasekaran, J., Kumar, S., 1994. Hourly Diffuse Fraction Correlation at a Tropical Location. Solar Energy 53 (6), 505-510.

[9] Chow, C., Urquhart, B., Lave, M., Dominguez, A., Kleissl, J., Shields, J., 2011. Intra-hour Forecasting with a Total Sky Imager at the UC San Diego Solar Energy Testbed. Solar Energy 85 (11), 2881-2893. 
[10] De Miguel, A., Bilbao, J., Aguiar, R., Kambezidis, H., Negro, E., Jan. 2001. Diffuse Solar Irradiation Model Evaluation in the North Mediterranean Belt Area. Solar Energy 70 (2), 143-153.

[11] Dervishi, S., Mahdavi, A., Jun. 2012. Computing Diffuse Fraction of Global Horizontal Solar Radiation: A Model Comparison. Solar Energy 86 (6), 1796-1802.

[12] Engerer, N. A., Mills, F. P., 2014. KPV: A Clear-Sky Index for Photovoltaics . Solar Energy 105 (July), 679-693.

[13] Engerer, N. A., Mills, F. P., 2015. Validating Nine Clear-Sky Radiation Models in Australia. Solar Energy (Submitted).

URL http://nickengerer.org

[14] Erbs, D., Klein, S., Duffle, J., 1982. Estimation of the Diffuse Radiation Fraction for Hourly, Daily and Monthly-Average Global Radiation. Solar Energy 28 (4), 293-302.

[15] Forgan, B., 1996. A New Method for Calibrating Reference and Field Pyranometers. Journal of Atmospheric and Oceanic Technology 13, 638-645.

[16] Gueymard, C., Mar. 2008. REST2: High-Performance Solar Radiation Model for Cloudless-Sky irradiance, Illuminance, and Photosynthetically Active Radiation: Validation with a Benchmark Dataset. Solar Energy $82(3), 272-285$.

[17] Gueymard, C., Mar. 2009. Direct and Indirect Uncertainties in the Prediction of Tilted Irradiance for Solar Engineering Applications. Solar Energy $83(3), 432-444$.

[18] Gueymard, C., 2010. Progress in Direct Irradiance Modeling and Validation. In: Solar 2010 Conference Proceedings.

[19] Gueymard, C., Myers, D., 2008. Validation and Ranking Methodologies for Solar Radiation Models. In: Modeling Solar Radiation at the Earth's Surface. Springer, pp. 479-509.

[20] Hawlader, M. N. A., Jan. 1984. Diffuse, Global and Extra-Terrestrial Solar Radiation for Singapore. International Journal of Ambient Energy 5 (1), $31-38$.

[21] Hinkley, J. T., Hayward, J. A., Curtin, B., Wonhas, A., Boyd, R., Grima, C., Tadros, A., Hall, R., Naicker, K., Sep. 2013. An Analysis of the Costs and Opportunities for Concentrating Solar Power in Australia. Renewable Energy 57, 653-661.

[22] Ineichen, P., Jun. 2008. Comparison and Validation of Three Globalto-Beam Irradiance Models against Ground Measurements. Solar Energy $82(6), 501-512$. 
[23] Iqbal, M., 1980. Prediction of Hourly Diffuse Solar Radiation from Measured Hourly Global Radiation on Horizontal Surface. Solar Energy 24, 491-503.

[24] Jacovides, C. P., Tymvios, F. S., Assimakopoulos, V. D., Kaltsounides, N. A., Dec. 2006. Comparative Study of Various Correlations in Estimating Hourly Diffuse Fraction of Global Solar Radiation. Renewable Energy 31 (15), 2492-2504.

[25] Karatasou, S., 2003. Analysis of Experimental Data on Diffuse Solar Radiation in Athens, Greece, for Building Applications. International Journal of Sustainable Energy 23 (1-2), 1-11.

[26] Kasten, F., 1980. A Simple Parameteriziation of the Pyrheliometric Formula for Determining the Linke Turbidity Factor. Meteoro. Rdsch. 33, 124127.

[27] Lam, J. C., Li, D. H., Nov. 1996. Correlation between Global Solar Radiation and its Direct and Diffuse Components. Building and Environment $31(6), 527-535$.

[28] Lanini, F., Wunderle, S., Lehning, M., Fierz, C., 2010. Division of Global Radiation into Direct Radiation and Diffuse Radiation. M.s., University of Bern.

[29] Liu, B., Jordan, R., 1960. The Interrelationship and Characteristic Distribution of Direct, Diffuse and Total Solar Radiation. Solar Energy 4 (3), $1-19$.

[30] Long, C. N., Shi, Y., 2006. The QCRad Value Added Product : Surface Radiation Measurement Quality Control Testing, Including Climatology Configurable Limits. Tech. Rep. ARM TR-074, Atmospheric Radiation Measurement Program.

[31] Louche, A., Poggi, P., Simonnot, G., Sanguinaires, R., 1991. Correlations for Direct Normal and Global Horizontal Irradiation on a French Mediterranean Site. Solar Energy 46 (4), 261-266.

[32] Luoma, J., Kleissl, J., Murray, K., Jan. 2012. Optimal Inverter Sizing Considering Cloud Enhancement. Solar Energy 86 (1), 421-429.

[33] Maxwell, E., 1987. A Quasi-Physical Model for Converting Hourly Global Horizontal to Direct Normal Insolation. Tech. rep., Solar Energy Research Institute.

[34] Mohammad, A., Moradi, I., Ali, G., 2008. Evaluation of 12 Models to Estimate Hourly Diffuse Irradiation on Inclined Surfaces. Renewable Energy $33,1406-1412$. 
[35] Muneer, T., Munawwar, S., 2006. Improved Accuracy Models For Hourly Diffuse Solar Radiation. Journal of Solar Energy Engineering 128 (1), 104.

[36] Notton, G., Poggi, P., Cristofari, C., Aug. 2006. Predicting Hourly Solar Irradiation on Inclined Surfaces Based on the Horizontal Measurements: Performances of the Association of Well-Known Mathematical Models. Energy Conversion and Management 47 (13-14), 1816-1829.

[37] Oliveira, A., Escobedo, J., Machado, A., Soares, J., 2002. Correlation Models of Diffuse Solar-Radiation Applied to the City of Sao Paulo, Brazil. Applied Energy 71, 59-73.

[38] Orgill, J., Hollands, K., Jan. 1977. Correlation Equation for Hourly Diffuse Radiation on a Horizontal Surface. Solar Energy 19 (4), 357-359.

[39] Paltridge, G., Proctor, D., 1976. Monthly Mean Solar Radiation Statistics for Australia. Solar Energy 18 (3), 235-243.

[40] Peel, M. C., Finlayson, B. L., McMahon, T. A., 2007. Updated World Map of the Köppen-Geiger Climate Classification. Hydrology and Earth System Sciences 11 (5), 1633-1644.

[41] Perez, R., Ineichen, P., Maxwell, E., Seals, R., Zelenka, A., 1992. Dynamic Global-to-Direct Irradiance Conversion Models. Tech. rep., ASHRAE.

[42] Perez, R., Seals, R., Zelenka, A., Ineichen, P., Jan. 1990. Climatic Evaluation of Models That Predict Hourly Direct Irradiance from Hourly Global Irradiance: Prospects for Performance Improvements. Solar Energy 44 (2), 99-108.

[43] Posadillo, R., López Luque, R., Feb. 2009. Hourly Distributions of the Diffuse Fraction of Global Solar Irradiation in Córdoba (Spain). Energy Conversion and Management 50 (2), 223-231.

[44] Randall, C., Whitson, M., 1977. Monthly Insolation and Meteorological Databases Including Improved Direct Insolation Estimates. Tech. rep., Aerospace Corporation, El Segundo, CA.

[45] Reindl, D., Beckman, W., Duffie, J., 1990. Diffuse Fraction Correlations. Solar Energy 45 (1), 1-7.

[46] Reindl, D., Beckman, W., Duffie, J., Jan. 1990. Evaluation of Hourly Tilted Surface Radiation Models. Solar Energy 45 (1), 9-17.

[47] Reno, M., Hansen, C., Stein, J., 2012. Global Horizontal Irradiance Clear Sky Models: Implementation and Analysis. Tech. Rep. March, Sandia National Laboratories.

[48] Ridley, B., Boland, J., Lauret, P., Feb. 2010. Modelling of Diffuse Solar Fraction with Multiple Predictors. Renewable Energy 35 (2), 478-483. 
[49] Rigollier, C., Lefevre, M., Wald, L., 2001. Heliosat version 2. Integration and Exploitation of Networked Solar Radiation Databases for Environment Monitoring (SoDa Project). Tech. rep.

URL http://soda-is.com

[50] Ruiz-Arias, J., Alsamamra, H., Tovar-Pescador, J., Pozo-Vázquez, D., May 2010. Proposal of a Regressive Model for the Hourly Diffuse Solar Radiation under All Sky Conditions. Energy Conversion and Management 51 (5), 881893.

[51] Skartveit, A., Olseth, J., Jan. 1987. A Model for the Diffuse Fraction of Hourly Global Radiation. Solar Energy 38 (4), 271-274.

[52] Soares, J., Oliveira, A. P., Božnar, M. Z., Mlakar, P., Escobedo, J. a. F., Machado, A. J., Oct. 2004. Modeling Hourly Diffuse Solar-Radiation in the City of São Paulo Using a Neural-Network Technique. Applied Energy 79 (2), 201-214.

[53] Spencer, J., Jan. 1982. A Comparison of Methods for Estimating Hourly Diffuse Solar Radiation from Global Solar Radiation. Solar Energy 29 (1), 19-32.

[54] Spitters, C., Toussaint, H., Goudriaan, J., 1986. Separating the Diffuse and Direct Component of Global Radiation and its Implications for Modeling Canopy Photosynthesis Part I: Components of Incoming Radiation. Agricultural and Forest Meteorology 38, 217-229.

[55] Stoffel, T., Reda, I., 2008. NREL Pyrheliometer Comparisons. Tech. rep., U.S. National Renewable Energy Laboratory, Golden, CO.

[56] Vazquez, M., Ruiz, V., Perez, R., 1991. The Roles of Scattering, Absorption, and Air Mass on the Diffuse-to-Global Correlations. Solar Energy 47 (3), 181-188.

[57] Vignola, F., McDaniels, D., 1986. Beam-Global Correlations in the Pacific Northwest. Solar Energy 26 (5), 409-418. 